PACS: 47.32.-y, 47.65.Md, 05.45.-a

\title{
NONLINEAR DYNAMO IN OBLIQUELY ROTATING STRATIFIED ELECTROCONDUCTIVE FLUID IN AN UNIFORMLY MAGNETIC FIELD
}

\author{
(DDichael I. Kopp ${ }^{\text {a*, }}$, Anatoly V. Tur ${ }^{\mathrm{c}}$, Konstantin N. Kulik ${ }^{\mathrm{a}}$, \\ (iD) Volodymyr V. Yanovsky ${ }^{\mathrm{a}, \mathrm{b}}$ * \\ ${ }^{a}$ Institute for Single Cristals, Nat. Academy of Science Ukraine, \\ Nauky Ave. 60, Kharkiv 31001, Ukraine \\ ${ }^{b}$ V.N. Karazin Kharkiv National University \\ 4, Svoboda Sq., Kharkiv, 61022, Ukraine \\ ${ }^{c}$ Universite Toulouse [UPS], CNRS, Institute of Research for Astrophysics and Planetology \\ 9 avenue du Colonel Roche, BP 44346, 31028 Toulouse Cedex 4, France \\ *Corresponding Author: michaelkopp0165@gmail.com \\ Received December 20, 2018; revised January 22, 2020; accepted January 28, 2020
}

\begin{abstract}
In this paper, we investigated a new large-scale instability that arises in an obliquely rotating convective electrically conducting fluid in an external uniform magnetic field with a small-scale external force with zero helicity. This force excites small-scale velocity oscillations with a small Reynolds number. Using the method of multiscale asymptotic expansions, we obtain the nonlinear equations for vortex and magnetic disturbances in the third order of the Reynolds number. It is shown that the combined effects of the Coriolis force and the small external forces in a rotating conducting fluid possible large-scale instability. The linear stage of the magnetovortex dynamo arising as a result of instabilities of $\alpha$-effect type is investigated. The mechanism of amplification of large-scale vortex disturbances due to the development of the hydrodynamic $\alpha$-effect taking into account the temperature stratification of the medium is studied. It was shown that a «weak» external magnetic field contributes to the generation of large-scale vortex and magnetic perturbations, while a «strong» external magnetic field suppresses the generation of magnetic-vortex perturbations. Numerical methods have been used to find stationary solutions of the equations of a nonlinear magneto-vortex dynamo in the form of localized chaotic structures in two cases when there is no external uniform magnetic field and when it is present.

KEY WORDS: equations of magnetic hydrodynamics in Boussinesq approximation, Coriolis force, multi-scale asymptotic expansions, small-scale non-helical turbulence, $\alpha$-effect, chaotic structures
\end{abstract}

As is known, the problems of generation of magnetic fields of planets, stars, galaxies and other space objects are studied within the framework of dynamo theory. For the first time, the term «dynamo» in connection with the generation of magnetic fields is introduced by Larmor [1]. In his opinion, hydrodynamic motion of an electroconductive fluid could engender a magnetic field by acting as the dynamo. In the linear theory or kinematic dynamo with the small magnetic energy compared to the medium motion kinetic energy, the magnetic forces hardly influence the medium flow. Currently, the kinematic theory of dynamo is practically built [2-11]. In this theory a significant role belongs to rotational motion of space bodies which generates various waves (e.g. Rossby or inertial waves ) and vortex motions (geostrophic, etc. [12-19]). In particular, under the influence of the Coriolis force the initial mirror-symmetric turbulence turns into helical one characterized by breakdown of the mirror symmetry of the turbulent fluid motion. The invariant $J_{s}=\overline{\vec{v} r o t \vec{v}}$ is the important topological characteristic of helical turbulence. It measures the knottedness of vortex field force lines [20]. It was shown in [21] that the generation of large-scale field occurs under the action of turbulent e.m.f. proportional to the mean magnetic field $\vec{\varepsilon}=\alpha \vec{H}$. Coefficient $\alpha$ is proportional to the mean helicity of the velocity field $\alpha \sim \overline{\vec{v} r o t \vec{v}}$ and is so called $\alpha$-effect. The generation properties of helical turbulence were considered not only in magnetic hydrodynamics or in electroconductive media, but in conventional hydrodynamics as well. For the first time the hypothesis that helical turbulence may generate largescale vortices was reported in [22]. It was based on the formal similarity of the equations of magnetic field induction $\vec{H}$ and those for vorticity $\vec{\omega}=\operatorname{rot} \vec{v}$.

However, as proved in [23], the $\alpha$-effect cannot occur in an incompressible turbulent fluid because of the symmetry of the Reynolds stress tensor in the averaged Navier-Stokes equations. Thus, for the appearance of the hydrodynamic $\alpha$-effect, one helicity of turbulence is not enough, other factors of symmetry breaking of turbulent flow are needed. As shown in [24] and [25], these factors are compressibility and temperature gradient in gravitational field, respectively. The effect of generation of large-scale vortex structures (LSVS) by helical turbulence is called vortex dynamo. The vortex dynamo mechanisms were developed with reference to the turbulent atmosphere and ocean. The theory of convective vortex dynamo was built in [25-31].

According to this theory, helical turbulence gives rise to a large-scale instability leading to the formation of a convective cell interpreted as a huge vortex of tropical cyclone type. There are many papers which deal with LSVS (C) Michael I. Kopp, Anatoly V. Tur, Konstantin N. Kulik, Volodymyr V. Yanovsky, 2020 
generation and take into account the effects of the rotation [32-37]. Just another $\alpha$-effect is reported in [38], where turbulent fluid motion is modelled by means of an external small-scale force $\vec{F}_{0}$. This model is characterized by parity violation (at zero helicity: $\vec{F}_{0} r o t \vec{F}_{0}=0$ ). The effect of generation of large-scale disturbances by such a force is called anisotropic kinetic $\alpha$-effect, or AKA-effect [38]. The mentioned paper consideres the large-scale instability in an incompressible fluid by means of the method of asymptotic multi-scale expansions. This method uses the Reynolds number $R=\frac{v_{0} t_{0}}{\lambda_{0}} \ll 1$ as a small parameter for small-scale pulsations of the velocity $v_{0}$ caused by the smallscale force. In real situations, because of the low kinematic viscosity, the Reynolds number number is large. However, in reality, the small scale turbulence generates turbulent viscosity. The Reynolds number calculated with this turbulent viscosity is not large $R=\frac{v_{0} t_{0}}{\lambda_{0}} \ll 1$. For this reason, the theory presented below can be applied approximately to real objects.

It is evident that applicability of kinematic theory of magnetic and vortex dynamos is limited. After a certain time, the intensified fields (vortex and magnetic ones) begin to affect the flows. In this case the behavior of the magnetic field and the motion of the meduim must be considered self-consistently, i.e. in the frame of the nonlinear theory. The observed magnetic fields of real objects exist probably just in nonlinear mode. So, the nonlinear theory [39] appears to be very important. In this paper the nonlinear theory of magnetic dynamo is based on generalization of the theory of mean field (see e.g. [7]) taking into account the nonlinear effects.

However, the theory of mean field does not allow us to determine the principal order at which the instability occurs from the whole hierarchy of perturbations. Therefore, an alternative for construction of a nonlinear dynamo theory is the method of multi-scale asymptotic expansions [38]. This method allows to construct the nonlinear theories of vortex dynamo for compressible media [40-41], as well as for convective media with a helical external force [30-31]. The asymptotic multi-scale method is used to reveal large-scale instability in the thermally stratified conductive medium in the case of helicity of small-scale velocity and magnetic fields [42-43]. Development of this large-scale instability in a convective electroconductive medium engenders the generation of both vortex and magnetic fields as well. Self-consistent or nonlinear theory of magneto-vortex dynamo in a convective electroconductive medium with small-scale helicity was built in [43].

In this work, the possibility of the formation of stationary chaotic large-scale structures in magnetic and vortex fields was shown for the first time. The particular case of the formation of large-scale stationary magnetic structures was also considered in this work. These structures were classified as stationary solutions of three types: nonlinear waves, solitons and kinks. Qualitative estimations of the linear stage [42] for solar conditions allow to set a good agreement of the characteristic scales and times of the formed hydrodynamic structures with those of the structures found experimentally [44].

In the above-mentioned papers helical turbulence was considered as a priori known, or the problem of its generation was examined independently [45]. The question naturally arises about the possibility of generation of large-scale vortices (hydrodynamic and magnetic) in rotating media under the action of a small-scale force with zero helicity $\vec{F}_{0} \operatorname{rot} \vec{F}_{0}=0$. The example of LSVS generation in a rotating incompressible fluid is found in [46]. The development of this large-scale instability in obliquely rotating fluid gives rise to nonlinear large-scale helical structures of Beltrami vortex type, or to localized kinks with internal helical structure. In [47] the new hydrodynamic $\alpha$-effect found in [46] was generalized to the case of electroconductive fluid. The corresponding large-scale instability leads to the generation of LSVS and magnetic fields. The nonlinear stage of this instability gives chaotic localized vortices and magneic structures. As is known [48-49], a large-scale motion caused by nonuniform heating in a gravitation field (free convection) exists in convective zones of the Sun and other stars, as well as in the core of the Earth and other planets. The convection in which the rotation axes of the medium and uniform magnetic field coincide with the direction of gravitation vector, was studied in detail in [49]. However, for astrophysical problems it is important to consider the case when the directions of the rotation axes and of magnetic fields are perpendicular, or do not coincide with each other. The role of azimuthal magnetic field significantly increases for convective fluid layers located in the equatorial region of the rotating object. As known from the theory of magnetic dynamo [2-7], the toroidal magnetic field in the Earth's crust and in the atmosphere of the Sun exceeds the poloidal magnetic field.

The aim of this work is to study generation and nonlinear evolution of vortices and magnetic fields in a rotating stratified electoconductive fluid in an external uniform magnetic field under the action of the nonhelical force $\vec{F}_{0}$. Suppose that the vector of angular rotation velocity $\vec{\Omega}$ is deviated from the vertical direction $O Z$, and the vector of the external magnetic field $\bar{B}$ is located in the horizontal plane XOY perpendicular to the direction of the gravity force $\vec{g}$ (Fig. 1). This geometry is most suitable for the description of dynamo processes in rotating space objects. The results obtained in the present work can be applied for various astrophysical problems. 

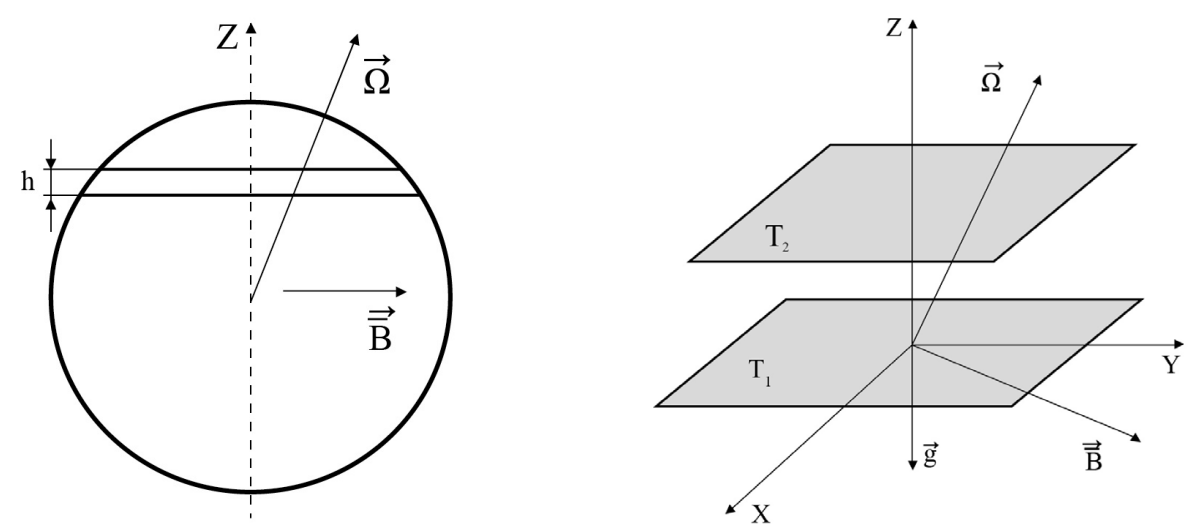

Fig.1. Shows schematically a thin layer of rotating electroconductive fluid of astrophysical object. For the general case the angular velocity $\vec{\Omega}$ is inclined to the plane $(X, Y)$ where the induction vector $\bar{B}$ of uniform magnetic filed is located. The gradient of equilibrium temperature is directed vertically downwards: $T_{1}>T_{2}$ - heating from below.

\section{BASIC EQUATIONS AND FORMULATION OF THE PROBLEM}

Consider the dynamics of perturbed state of the electroconductive fluid located in the constant gravitation $\vec{g}$ and magnetic $\overrightarrow{\bar{B}}$ fields with the constant temperature gradient $\nabla \bar{T}$ in the system of rotating coordinates:

$$
\begin{gathered}
\frac{\partial v_{i}}{\partial t}+v_{k} \frac{\partial v_{i}}{\partial x_{k}}=v \frac{\partial^{2} v_{i}}{\partial x_{k}^{2}}-\frac{1}{\bar{\rho}} \frac{\partial P}{\partial x_{i}}+2 \varepsilon_{i j k} v_{j} \Omega_{k}+\frac{\varepsilon_{i j k} \varepsilon_{j m l}}{4 \pi \bar{\rho}} \frac{\partial B_{l}}{\partial x_{m}}\left(B_{k}+\bar{B}_{k}\right)+ \\
+g e_{i} \beta \Theta+F_{0}^{i} \\
\frac{\partial B_{i}}{\partial t}=\varepsilon_{i j k} \varepsilon_{k n p} \frac{\partial}{\partial x_{j}}\left(v_{n}\left(B_{p}+\bar{B}_{p}\right)\right)+v_{m} \frac{\partial^{2} B_{i}}{\partial x_{k}^{2}} \\
\frac{\partial \Theta}{\partial t}+v_{k} \frac{\partial \Theta}{\partial x_{k}}-A e_{k} v_{k}=\chi \frac{\partial^{2} \Theta}{\partial x_{k}^{2}} \\
\frac{\partial v_{i}}{\partial x_{i}}=\frac{\partial B_{i}}{\partial x_{i}}=0
\end{gathered}
$$

Here $v_{i}, P, B_{i}, \Theta$ are the perturbations of velocity, pressure, magnetic field induction and fluid temperature $(i=x, y, z) . \bar{B}_{i}=$ const is the induction of the external homogeneous magnetic field; $\bar{\rho}$ is the equilibrium density of the medium $\bar{\rho}=$ const $, v, \chi$ are fluid viscosity and thermal conductivity coefficients respectively, $v_{m}=\frac{c^{2}}{4 \pi \sigma_{c}}$ is the magnetic viscosity coefficient. $\sigma_{c}$ is coefficient of electrical conductivity of the medium and $\beta$ is thermal expansion coefficient. The system of magnetic hydrodynamic eqs. (1)-(4) is written in the Boussinesq approximation [48] and describes the evolution of disturbances relative to the equilibrium state given by the constant temperature gradient $\nabla \bar{T}=-A \vec{e} \quad(A>0)$ and the hydrostatic pressure: $\nabla\left(\bar{P}+\frac{\bar{B}^{2}}{8 \pi}\right)=\bar{\rho} \vec{g}$. Here we neglect the centrifugal forces, since the condition $g \gg \Omega^{2} r$, where $r$ is the characteristic radius of fluid rotation, is satisfied. Now let us formulate the problem with the geometry shown in Fig. 1. Consider a thin layer (with the thickness $h$ ) of a rotating electoconductive fluid in which the lower and the upper surfaces have the temperatures $T_{1}$ and $T_{2}$, respectively. We suppose $T_{1}>T_{2}$, i.e. heating from below. In this case the direction of the temperature gradient $\nabla \bar{T}=\vec{A}$ coincides with the direction of the gravitation field $\vec{g}=-g \vec{e}_{z}$. Here $\vec{e}=(0,0,1)$ is the unit vector in the direction of the axis $Z$. The temperature profile $\bar{T}$ depends linearly on the vertical coordinate $z: \bar{T}(z)=T_{1}-\frac{T_{1}-T_{2}}{h} \cdot z$. The vector of angular rotation velocity $\vec{\Omega}=\left(\Omega_{1}, \Omega_{2}, \Omega_{3}\right)$ is constant (solid-body rotation) and inclined with respect to the plane $(X, Y)$ where is the vector of homogeneous magnetic field $\overrightarrow{\bar{B}}=\left(\bar{B}_{1}, \bar{B}_{2}, 0\right)$. 
Eq. (1) contains the external force $\vec{F}_{0}$. It models the source of external excitation in the medium of small-scale and high-frequency fluctuations of the velocity field $\vec{v}_{0}$ with the small Reynolds number $R=\frac{v_{0} t_{0}}{\lambda_{0}} \ll 1$. Here we will consider the non-helical external force $\vec{F}_{0}$ with the following properties:

$$
\operatorname{div} \vec{F}_{0}=0, \vec{F}_{0} \operatorname{rot} \vec{F}_{0}=0, \operatorname{rot} \vec{F}_{0} \neq 0, \vec{F}_{0}=f_{0} \vec{F}_{0}\left(\frac{x}{\lambda_{0}} ; \frac{t}{t_{0}}\right)
$$

where $\lambda_{0}$ is the characteristic scale, $t_{0}$ is the characteristic time, $f_{0}$ is the characteristic amplitude of the external force. Now choose the external force in a rotating coordinate system in the form:

$$
\begin{gathered}
F_{0}^{z}=0, \vec{F}_{0}=f_{0}\left(\vec{i} \cos \varphi_{2}+\vec{j} \cos \varphi_{1}\right), \\
\varphi_{1}=\vec{\kappa}_{1} \vec{x}-\omega_{0} t, \quad \varphi_{2}=\vec{\kappa}_{2} \vec{x}-\omega_{0} t, \\
\vec{\kappa}_{1}=\kappa_{0}(1,0,0), \vec{\kappa}_{2}=\kappa_{0}(0,1,0) .
\end{gathered}
$$

It is evident that this external force satisfies all the conditions (5). Let us consider the dimensionless variables in eqs. (1)-(4). For convenience we keep the same notation as for dimensional variables:

$$
\begin{gathered}
\vec{x} \rightarrow \frac{\vec{x}}{\lambda_{0}}, \quad t \rightarrow \frac{t}{t_{0}}, \quad \vec{v} \rightarrow \frac{\vec{v}}{v_{0}}, \quad \vec{F}_{0} \rightarrow \frac{\vec{F}_{0}}{f_{0}}, \\
\vec{B} \rightarrow \frac{\vec{B}}{B_{0}}, \quad \overline{\vec{B}} \rightarrow \frac{\overline{\vec{B}}}{B_{0}}, \quad \Theta \rightarrow \frac{\Theta}{\lambda_{0} A}, \\
t_{0}=\frac{\lambda_{0}^{2}}{v}, \quad f_{0}=\frac{v_{0} v}{\lambda_{0}^{2}}, \quad P \rightarrow \frac{P}{P_{0}}, \quad P_{0}=\bar{\rho} \frac{v v_{0}}{\lambda_{0}} .
\end{gathered}
$$

Here $v_{0}, B_{0}, P_{0}$ are the characteristic values of small-scale pulsations of the velocity, magnetic field and pressure. In the dimensionless variables eqs.(1)-(3) take the form:

$$
\begin{gathered}
\frac{\partial v_{i}}{\partial t}+R v_{k} \frac{\partial v_{i}}{\partial x_{k}}=\frac{\partial^{2} v_{i}}{\partial x_{k}^{2}}-\frac{\partial P}{\partial x_{i}}+\varepsilon_{i j k} v_{j} D_{k}+\frac{Q}{R P m} \varepsilon_{i j k} \varepsilon_{j m l} \frac{\partial B_{l}}{\partial x_{m}}\left(B_{k}+\bar{B}_{k}\right)+ \\
+e_{i} \frac{R a}{R P r} \Theta+F_{0}^{i} \\
\frac{\partial B_{i}}{\partial t}-P m^{-1} \frac{\partial^{2} B_{i}}{\partial x_{k}^{2}}=R \varepsilon_{i j k} \varepsilon_{k n p} \frac{\partial}{\partial x_{j}}\left(v_{n}\left(B_{p}+\bar{B}_{p}\right)\right) \\
\frac{\partial \Theta}{\partial t}+R v_{k} \frac{\partial \Theta}{\partial x_{k}}-\operatorname{Re}_{k} v_{k}=\operatorname{Pr}^{-1} \frac{\partial^{2} \Theta}{\partial x_{k}^{2}}
\end{gathered}
$$

Whithe new temperature $\Theta \rightarrow \Theta / R$ and magnetic field $B \rightarrow B / R$ we obtain finally:

$$
\begin{gathered}
\frac{\partial v_{i}}{\partial t}+R v_{k} \frac{\partial v_{i}}{\partial x_{k}}=\frac{\partial^{2} v_{i}}{\partial x_{k}^{2}}-\frac{\partial P}{\partial x_{i}}+\varepsilon_{i j k} v_{j} D_{k}+R \tilde{Q} \varepsilon_{i j k} \varepsilon_{j m l} \frac{\partial B_{l}}{\partial x_{m}} B_{k}+ \\
+\tilde{Q} \varepsilon_{i j k} \varepsilon_{j m l} \frac{\partial B_{l}}{\partial x_{m}} \bar{B}_{k}+e_{i} \operatorname{Ra} \Theta+F_{0}^{i} \\
\frac{\partial B_{i}}{\partial t}-P m^{-1} \frac{\partial^{2} B_{i}}{\partial x_{k}^{2}}=R \varepsilon_{i j k} \varepsilon_{k n p} \frac{\partial}{\partial x_{j}}\left(v_{n} B_{p}\right)+\varepsilon_{i j k} \varepsilon_{k n p} \frac{\partial}{\partial x_{j}}\left(v_{n} \bar{B}_{p}\right) \\
\frac{\partial \Theta}{\partial t}-\operatorname{Pr}^{-1} \frac{\partial^{2} \Theta}{\partial x_{k}^{2}}=-R v_{k} \frac{\partial \Theta}{\partial x_{k}}+e_{k} v_{k} \\
\frac{\partial v_{i}}{\partial x_{i}}=\frac{\partial B_{i}}{\partial x_{i}}=0
\end{gathered}
$$


Here we use the following dimensionless parameters: $\tilde{R a}=\frac{R a}{P r}, R a=\frac{g \beta A \lambda_{0}^{4}}{v \chi}$ is the Rayleigh number in the scale $\lambda_{0} ; D_{i}=\frac{2 \Omega_{i} \lambda_{0}^{2}}{v}$ - the rotation parameter in the scale $\lambda_{0}(i=1,2,3)$ connected with the Taylor number $T a_{i}=D_{i}^{2} ; \tilde{Q}=\frac{Q}{P m}, Q=\frac{\sigma_{c} B_{0}^{2} \lambda_{0}^{2}}{c^{2} \bar{\rho} v}-$ the Chandrasekhar number, $P m=\frac{v}{v_{m}}$ - the magnetic Prandtl number, $\operatorname{Pr}=\frac{v}{\chi}-$ the Prandtl number. The small parameter of asymptotic expansion is the Reynolds number $R=\frac{v_{0} t_{0}}{\lambda_{0}} \ll 1$ and the parameters $D, \tilde{Q}$ and $\widetilde{R a}$ are arbitrary and do not affect the scheme of asymptotic expansion. The presence of the small parameter $(R \ll 1)$ in the system of eqs. (10)-(13) allows us to apply the theory of multi-scale asymptotic expansions (see e.g. [30-31], [38]). In contrast to the theory of mean field [2-7] we can consistently see the dynamics of disturbances for different spatial and temporal scales in each order by $R$. In particular, in the zero order of $R$, smallscale and high-frequency oscillations of the velocity $\vec{v}_{0}$ are excited by the external force $\vec{F}_{0}$ acting at the equilibrium state. Obviously, the dynamics of small-scale fields depends on external factors such as rotation and stratification of the medium, magnetic and gravitation fields, etc. These oscillations are characterized by zero average values. Nevertheless, the nonlinear interactions in some orders of the perturbation theory give rise to the terms which do not vanish at averaging. In the next section, we consider in detail how to find the solvability conditions for the multi-scale asymptotic expansion, which define the evolution equations for large-scale perturbations.

\section{EQUATIONS FOR LARGE-SCALE FIELDS}

In accordance with the method of construction of asymptotic equations [30-31], [38] let us present spatial and temporal derivatives in eqs. (10)-(13) in the form of the asymptotic expansion:

$$
\frac{\partial}{\partial t} \rightarrow \partial_{t}+R^{4} \partial_{T}, \frac{\partial}{\partial x_{i}} \rightarrow \partial_{i}+R^{2} \nabla_{i}
$$

where $\partial_{i}$ and $\partial_{t}$ are the derivatives with respect to the fast variables $x_{0}=\left(\vec{x}_{0}, t_{0}\right)$, whereas $\nabla_{i}$ and $\partial_{T}$ are the derivatives with respect to the slow variables $X=(\vec{X}, T)$. The variables $x_{0}$ and $X$ may be referred to as small- and large-scale variables, accordingly. While constructing the nonlinear theory we present the variables $\vec{V}, \vec{B}, P$ in the form of the asymptotic series:

$$
\begin{gathered}
\vec{V}(\vec{x}, t)=\frac{1}{R} \vec{W}_{-1}(X)+\vec{v}_{0}\left(x_{0}\right)+R \vec{v}_{1}+R^{2} \vec{v}_{2}+R^{3} \vec{v}_{3}+\cdots \\
\vec{B}(\vec{x}, t)=\frac{1}{R} \vec{B}_{-1}(X)+\vec{B}_{0}\left(x_{0}\right)+R \vec{B}_{1}+R^{2} \vec{B}_{2}+R^{3} \vec{B}_{3}+\cdots \\
\Theta(\vec{x}, t)=\frac{1}{R} T_{-1}(X)+T_{0}\left(x_{0}\right)+R T_{1}+R^{2} T_{2}+R^{3} T_{3}+\cdots \\
P(x)=\frac{1}{R^{3}} P_{-3}+\frac{1}{R^{2}} P_{-2}+\frac{1}{R} P_{-1}+P_{0}\left(x_{0}\right)+R\left(P_{1}+\bar{P}_{1}(X)\right)+R^{2} P_{2}+R^{3} P_{3}+\cdots
\end{gathered}
$$

Let us substitute the expansions (14)-(15) into the system of eqs. (10)-(13). Then we select the terms of the same orders of $R$ up to the degree $R^{3}$ and obtain the equations of multi-scale asymptotic expansion. The algebraic structure of the asymptotic expansion of eqs. (10)-(13) of different orders in $R$ is presented in Appendix A. Here it is shown that the basic secular equations, i.e. those for large-scale fields, are obtained in the order $R^{3}$

$$
\begin{gathered}
\partial_{t} W_{-1}^{i}-\nabla_{k}^{2} W_{-1}^{i}+\nabla_{k} \overline{\left(v_{0}^{k} v_{0}^{i}\right)}=-\nabla_{i} \bar{P}_{1}+\tilde{Q} \varepsilon_{i j k} \varepsilon_{j m l}\left(\nabla_{m} \overline{\left(B_{0}^{l} B_{0}^{k}\right)}\right) \\
\partial_{t} B_{-1}^{i}-P m^{-1} \nabla_{k}^{2} B_{-1}^{i}=\varepsilon_{i j k} \varepsilon_{k n p} \nabla_{j} \overline{\left(v_{0}^{n} B_{0}^{p}\right)} \\
\partial_{T} T_{-1}-P r^{-1} \nabla_{k}^{2} T_{-1}=-\nabla_{k}\left(\overline{v_{0}^{k} T_{0}}\right)
\end{gathered}
$$


Using the convolution of the tensors $\varepsilon_{i j k} \varepsilon_{j m l}=\delta_{k m} \delta_{i l}-\delta_{i m} \delta_{k l}, \varepsilon_{i j k} \varepsilon_{k n p}=\delta_{i n} \delta_{j p}-\delta_{i p} \delta_{j n}$ and the denotations $\vec{W}=\vec{W}_{-1}, \vec{H}=\vec{B}_{-1}$ obtain eqs. (16)-(17) in the following form:

$$
\begin{gathered}
\partial_{T} W_{i}-\nabla_{k}^{2} W_{i}+\nabla_{k} \overline{\left(v_{0}^{k} v_{0}^{i}\right)}=-\nabla_{i} \bar{P}_{1}+\tilde{Q}\left(\nabla_{k} \overline{\left(B_{0}^{i} B_{0}^{k}\right)}-\frac{\nabla_{i}}{2} \overline{\left(B_{0}^{k}\right)^{2}}\right) \\
\partial_{T} H_{i}-P m^{-1} \nabla_{k}^{2} H_{i}=\nabla_{j} \overline{\left(v_{0}^{i} B_{0}^{j}\right)}-\nabla_{j} \overline{\left(v_{0}^{j} B_{0}^{i}\right)}
\end{gathered}
$$

Eqs. (16)-(18) are supplemented with the secular equations derived in Appendix A:

$$
\begin{gathered}
-\nabla_{i} P_{-3}+\varepsilon_{i j k} W_{j} D_{k}+e_{i} \tilde{R a} T_{-1}=0, \quad W_{-1}^{z}=0, \\
W_{-1}^{k} \nabla_{k} W_{-1}^{i}=-\nabla_{i} P_{-1}+\tilde{Q} \varepsilon_{i j k} \varepsilon_{j m l}\left(\nabla_{m} B_{-1}^{l} B_{-1}^{k}+\nabla_{m} B_{-1}^{l} \overline{B_{k}}\right), \\
\varepsilon_{i j k} \varepsilon_{k n p}\left(\nabla_{j} W_{-1}^{n} B_{-1}^{p}+\nabla_{j} W_{-1}^{n} \overline{B_{p}}\right)=0, \\
W_{-1}^{k} \nabla_{k} T_{-1}=0, \quad \nabla_{i} W_{-1}^{i}=0, \quad \nabla_{i} B_{-1}^{i}=0 .
\end{gathered}
$$

To obtain the system of eqs. (16)-(18) which decribe the evolution of large-scale fields we have to reach the third order of the perturbation theory. This is a rather typical phenomenon when applying the method of multiscale expansions. As seen from eqs. (16)-(17), the large-scale temperature $T_{-1}$ does not influence the dynamics of the largescale field of the velocity $\vec{W}_{-1}$ and the magnetic field $\vec{B}_{-1}$, therefore let us confine ourselves to investigation of eqs. (16)-(17). These equations acquire a closed form after calculation of the correlation functions, i.e. the Reynolds stresses $\nabla_{k} \overline{\left(v_{0}^{k} v_{0}^{i}\right)}$, the Maxwell stresses $\nabla_{k} \overline{\left(B_{0}^{i} B_{0}^{k}\right)}$ and the turbulent e.m.f. $\mathcal{E}_{n}=\varepsilon_{n i j} \overline{v_{0}^{i} B_{0}^{j}}$. Calculation of these correlation functions is significantly simplified due to the «quasi-two-dimensional» approximation which is often used for description of large-scale vortices and magnetic fields in many astrophysical and geophysical problems. In the framework of this approximation we consider the large-scale derivative with respect to $Z$ as more important than horizontal derivatives, i.e.

$$
\nabla_{Z} \equiv \frac{\partial}{\partial Z} \gg \frac{\partial}{\partial X}, \frac{\partial}{\partial Y} .
$$

Therefore, the geometry of large-scale fields has the following form:

$$
\vec{W}=\left(W_{1}(Z), W_{2}(Z), 0\right), \vec{H}=\left(H_{1}(Z), H_{2}(Z), 0\right)
$$

For the «quasi-two-dimensional» problem the system of eqs. (14)-(15) is simplified:

$$
\begin{gathered}
\partial_{T} W_{1}-\nabla_{Z}^{2} W_{1}+\nabla_{Z} \overline{\left(v_{0}^{z} v_{0}^{x}\right)}=\tilde{Q} \nabla_{Z} \overline{\left(B_{0}^{z} B_{0}^{x}\right)} \\
\partial_{T} W_{2}-\nabla_{Z}^{2} W_{2}+\nabla_{Z} \overline{\left(v_{0}^{z} v_{0}^{y}\right)}=\tilde{Q} \nabla_{Z} \overline{\left(B_{0}^{z} B_{0}^{y}\right)} \\
\partial_{T} H_{1}-P m^{-1} \nabla_{Z}^{2} H_{1}=\nabla_{Z} \overline{\left(v_{0}^{x} B_{0}^{z}\right)}-\nabla_{Z} \overline{\left(v_{0}^{z} B_{0}^{x}\right)} \\
\partial_{T} H_{2}-P m^{-1} \nabla_{Z}^{2} H_{2}=\nabla_{Z} \overline{\left(v_{0}^{y} B_{0}^{z}\right)}-\nabla_{Z} \overline{\left(v_{0}^{z} B_{0}^{y}\right)} \\
\partial_{T} T_{-1}-P^{-1} \nabla_{Z}^{2} T_{-1}+\nabla_{Z}\left(\overline{v_{0}^{z} T_{0}}\right)=0
\end{gathered}
$$

To have eqs. (22) $-(26)$ in the closed form we use the solutions of the equations for small-scale fields in the zero order in $R$ obtained in Appendix B. Then it is necessary to calculate the correlators contained in the system (22)-(26). The technical aspect of this problem is considered in detail in Appendix C. The calculations performed here make possible to obtain the following closed equations for large-scale fields of the velocity $\left(W_{1}, W_{2}\right)$ and the magnetic fields $\left(H_{1}, H_{2}\right)$ :

$$
\partial_{T} W_{1}-\nabla_{Z}^{2} W_{1}+\nabla_{Z}\left(\alpha_{(2)} \cdot\left(1-W_{2}\right)\right)=0
$$




$$
\begin{aligned}
& \partial_{T} W_{2}-\nabla_{Z}^{2} W_{2}-\nabla_{Z}\left(\alpha_{(1)} \cdot\left(1-W_{1}\right)\right)=0 \\
& \partial_{T} H_{1}-P m^{-1} \nabla_{Z}^{2} H_{1}+\nabla_{Z}\left(\alpha_{H}^{(2)} \cdot H_{2}\right)=0 \\
& \partial_{T} H_{2}-P m^{-1} \nabla_{Z}^{2} H_{2}-\nabla_{Z}\left(\alpha_{H}^{(1)} \cdot H_{1}\right)=0
\end{aligned}
$$

where the nonlinear coefficients $\alpha_{(1)}, \alpha_{(2)}, \alpha_{H}^{(1)}, \alpha_{H}^{(2)}$ have the form:

$$
\begin{gathered}
\alpha_{(1)}=\frac{f_{0}^{2}}{2} \cdot \frac{D_{1} q_{1} Q_{1}\left(1-W_{1}\right)^{-1}}{4\left(1-W_{1}\right)^{2} q_{1}^{2} \tilde{Q}_{1}^{2}+\left[D_{1}^{2}+W_{1}\left(2-W_{1}\right)+\mu_{1}\right]^{2}+\xi_{1}}, \\
\alpha_{(2)}=\frac{f_{0}^{2}}{2} \cdot \frac{D_{2} q_{2} Q_{2}\left(1-W_{2}\right)^{-1}}{4\left(1-W_{2}\right)^{2} q_{2}^{2} \tilde{Q}_{2}^{2}+\left[D_{2}^{2}+W_{2}\left(2-W_{2}\right)+\mu_{2}\right]^{2}+\xi_{2}}, \\
\alpha_{H}^{(1)}=f_{0}^{2} \cdot \frac{D_{1}\left(1-W_{1}\right) P m \tilde{Q}_{1}\left(1+\bar{B}_{1} / H_{1}\right)}{\left(1+P m^{2}\left(1-W_{1}\right)^{2}\right) \cdot \tilde{\xi}_{1}}, \\
\alpha_{H}^{(2)}=f_{0}^{2} \cdot \frac{D_{2}\left(1-W_{2}\right) P m \tilde{Q}_{2}\left(1+\bar{B}_{2} / H_{2}\right)}{\left(1+P m^{2}\left(1-W_{2}\right)^{2}\right) \cdot \tilde{\xi}_{2}} .
\end{gathered}
$$

The expressions which denote $q_{1,2}, Q_{1,2}, \tilde{Q}_{1,2}, \mu_{1,2}, \sigma_{1,2}, \chi_{1,2}, \xi_{1,2}, \tilde{\xi}_{1,2}$ are also presented in Appendix C. The coefficients $\alpha_{(1)}, \alpha_{(2)}$ and $\alpha_{H}^{(1)}, \alpha_{H}^{(2)}$ correspond to the nonlinear HD $\alpha$-effect and the nonlinear MHD $\alpha$-effect, respectively. Thus, we have obtained the self-consistent system of nonlinear evolution equations for the large-scale perturbations of the velocity and magnetic field which further are called the equations of nonlinear magneto-vortex dynamo. It should be noted that the mechanism of dynamo «works» only due to the effect of rotation of the medium. If this rotation is absent $(\Omega=0)$, then the diffuse spreading of large-scale fields occurs. In the absence of heating $(\nabla \bar{T}=0)$ and of external magnetic field $(\bar{B}=0)$ eqs. (27)-(28) coincide with the results found in [47]. In the case of non-electroconductive fluid $(\sigma=0)$ with the temperature gradient $(\nabla \bar{T} \neq 0)$ we obtain the same results as in [50]. In the limit of non-electroconductive $(\sigma=0)$ and homogeneous fluid $(\nabla \bar{T}=0)$ we obtain the results of [46]. To study this dynamo model, it is necessary at first to consider the evolution of small perturbations and then to examine the nonlinear effects.

\section{LARGE-SCALE INSTABILITY}

Let us consider the behavior of small perturbations of the field of velocity $\left(W_{1}, W_{2}\right)$ and the magnetic fields $\left(H_{1}, H_{2}\right)$. Then expand the nonlinear coefficients $\alpha_{(1,2)}$ and $\alpha_{H}^{(1,2)}$ in eqs. (27)-(30) into the Taylor series with respect to the small values $\left(W_{1}, W_{2}\right),\left(H_{1}, H_{2}\right)$ :

$$
\begin{gathered}
\alpha_{(1,2)} \cdot\left(1-W_{1,2}\right) \approx \alpha_{0}^{(1,2)}-\alpha_{1,2}^{(H)} \cdot H_{1,2}-\alpha_{1,2}^{(W)} \cdot W_{1,2}, \alpha_{0}^{(1,2)}=\text { const }, \\
\alpha_{H}^{(1,2)} \cdot H_{1,2} \approx \alpha_{0 H}^{(1,2)}+\tilde{\alpha}_{H}^{(1,2)} \cdot H_{1,2}-\beta_{W}^{(1,2)} \cdot W_{1,2}, \alpha_{0 H}^{(1,2)}=\text { const. }
\end{gathered}
$$

After substituting (31) into eqs. (27)-(30) we obtain the linearized system of equations :

$$
\begin{aligned}
& \partial_{T} W_{1}-\nabla_{Z}^{2} W_{1}-\alpha_{2}^{(H)} \cdot \nabla_{Z} H_{2}-\alpha_{2}^{(W)} \cdot \nabla_{Z} W_{2}=0 \\
& \partial_{T} W_{2}-\nabla_{Z}^{2} W_{2}+\alpha_{1}^{(H)} \cdot \nabla_{Z} H_{1}+\alpha_{1}^{(W)} \cdot \nabla_{Z} W_{1}=0 \\
& \partial_{T} H_{1}-\nabla_{Z}^{2} H_{1}+\tilde{\alpha}_{H}^{(2)} \cdot \nabla_{Z} H_{2}-\beta_{W}^{(2)} \cdot \nabla_{Z} W_{2}=0 \\
& \partial_{T} H_{2}-\nabla_{Z}^{2} H_{2}-\tilde{\alpha}_{H}^{(1)} \cdot \nabla_{Z} H_{1}+\beta_{W}^{(1)} \cdot \nabla_{Z} W_{1}=0,
\end{aligned}
$$

where the constant coefficients $\alpha_{1,2}^{(H)}, \alpha_{1,2}^{(W)}, \tilde{\alpha}_{H}^{(1,2)}, \beta_{W}^{(1,2)}$ have the following form: 


$$
\begin{gathered}
\alpha_{1,2}^{(H)}=\frac{f_{0}^{2} D_{1,2}}{2} \cdot Q \bar{B}_{1,2}\left[\frac{(2-R a)\left(2-Q \bar{B}_{1,2}^{2}\right)\left(4\left(D_{1,2}^{2}-R a\right)+(R a+1)^{2}+7\right)}{4\left(4+\left(D_{1,2}^{2}-R a\right)^{2}\right)^{2}}+\frac{Q \bar{B}_{1,2}^{2}-2(R a-1)}{4\left(4+\left(D_{1,2}^{2}-R a\right)^{2}\right)}\right], \\
\alpha_{1,2}^{(W)}=\frac{f_{0}^{2} D_{1,2}}{2} \cdot\left[\frac{(2-R a)\left(2-Q \bar{B}_{1,2}^{2}\right)\left(D_{1,2}^{2}-R a-2\right)}{\left(4+\left(D_{1,2}^{2}-R a\right)^{2}\right)^{2}}+\frac{Q \bar{B}_{1,2}^{2}+R a\left(1-Q \bar{B}_{1,2}^{2}\right)}{2\left(4+\left(D_{1,2}^{2}-R a\right)^{2}\right)}\right], \\
\tilde{\alpha}_{H}^{(1,2)}=\frac{f_{0}^{2} D_{1,2}}{4} \cdot\left[\frac{2+R a-Q \bar{B}_{1,2}^{2}+\bar{B}_{1,2}(2+R a)}{4+\left(D_{1,2}^{2}-R a\right)^{2}}-\frac{\bar{B}_{1,2}(2+R a)\left(4\left(D_{1,2}^{2}-R a\right)+(R a+1)^{2}+7\right)}{\left(4+\left(D_{1,2}^{2}-R a\right)^{2}\right)^{2}}\right], \\
\beta_{W}^{(1,2)}=f_{0}^{2} \cdot \frac{D_{1,2} \bar{B}_{1,2}(2+R a)\left(D_{1,2}^{2}-R a-2\right)}{\left(4+\left(D_{1,2}^{2}-R a\right)^{2}\right)^{2}}-\frac{f_{0}^{2}}{4} \cdot \frac{D_{1,2} \bar{B}_{1,2} R a}{4+\left(D_{1,2}^{2}-R a\right)^{2}}
\end{gathered}
$$

To obtain the system of equations (32)-(35) we use a simplification when the equality of Prandtl numbers is equal to unity: $\operatorname{Pr}=P m=1$. As seen from eqs. (32)-(35), in the presence of external magnetic field the coefficients $\alpha_{1,2}^{(H)}$ and $\beta_{W}^{(1,2)}$ define the positive feedback in the self-consistent dynamics of the fields $W_{1,2}$ and $H_{1,2}$. Now let us find the solution of the linear system of eqs. (32)-(35) in the form of plane waves with the wave vector $\vec{K} \| O Z$ :

$$
\left(\begin{array}{l}
W_{1,2} \\
H_{1,2}
\end{array}\right)=\left(\begin{array}{c}
W_{1,2} \\
\mathscr{\Psi}_{1,2}
\end{array}\right) \exp (-i \omega T+i K Z)
$$

After substituting (40) into the system (32)-(35) we obtain the dispersion equation:

$$
\begin{gathered}
{\left[\left(K^{2}-i \omega\right)^{2}-K^{2}\left(\alpha_{1}^{(W)} \alpha_{2}^{(W)}+\alpha_{2}^{(H)} \beta_{W}^{(1)}\right)\right]\left[\left(K^{2}-i \omega\right)^{2}-K^{2}\left(\tilde{\alpha}_{H}^{(1)} \tilde{\alpha}_{H}^{(2)}+\alpha_{1}^{(H)} \beta_{W}^{(2)}\right)\right]+} \\
+K^{4}\left(\tilde{\alpha}_{H}^{(1)} \alpha_{2}^{(H)}-\alpha_{1}^{(H)} \alpha_{2}^{(W)}\right)\left(\alpha_{1}^{(W)} \beta_{W}^{(2)}-\tilde{\alpha}_{H}^{(2)} \beta_{W}^{(1)}\right)=0
\end{gathered}
$$

Analysis of dispersion equation (41) in the absence of external magnetic field $\bar{B}_{1,2}=0$

It is obvious that without external magnetic field $\bar{B}_{1,2}=0$ the coefficients $\alpha_{1,2}^{(H)}$ and $\beta_{W}^{(W)}$ vanish, and (41) breaks down into two independent equations:

$$
\left[\left(K^{2}-i \omega\right)^{2}-\alpha_{1}^{(W)} \alpha_{2}^{(W)} K^{2}\right]\left[\left(K^{2}-i \omega\right)^{2}-\tilde{\alpha}_{H}^{(1)} \tilde{\alpha}_{H}^{(2)} K^{2}\right]=0
$$

where the coefficients $\alpha_{1,2}^{(W)}, \tilde{\alpha}_{H}^{(1,2)}$ do not depend on $\bar{B}_{1,2}$. Dispersion eq. (42) corresponds to the physical situation when small perturbations of vortex and magnetic fields independently gain in intensity due to development of largescale instability such as $\alpha$-effect. Using the frequency $\omega=\omega_{0}+i \Gamma$ from eq. (42) we find:

$$
\begin{aligned}
& \Gamma_{1}=\operatorname{Im} \omega_{1}= \pm \sqrt{\alpha_{1}^{(W)} \alpha_{2}^{(W)}} K-K^{2} \\
& \Gamma_{2}=\operatorname{Im} \omega_{2}= \pm \sqrt{\tilde{\alpha}_{H}^{(1)} \tilde{\alpha}_{H}^{(2)}} K-K^{2}
\end{aligned}
$$

Solutions (43) show the instability at $\alpha_{1} \alpha_{2}>0$ for large-scale vortex perturbations with the maximum instability increment $\Gamma_{1 \max }=\frac{\alpha_{1} \alpha_{2}}{4}$ at the wave numbers $K_{1 \max }=\frac{\sqrt{\alpha_{1} \alpha_{2}}}{2}$. Similarly, for magnetic perturbations the instability increment $\Gamma_{2 \max }=\frac{\tilde{\alpha}_{H}^{(1)} \tilde{\alpha}_{H}^{(2)}}{4}$ reaches its maximum at the wave numbers $K_{2 \max }=\frac{\sqrt{\tilde{\alpha}_{H}^{(1)} \tilde{\alpha}_{H}^{(2)}}}{2}$. If $\alpha_{1} \alpha_{2}<0$ and 
$\tilde{\alpha}_{H}^{(1)} \tilde{\alpha}_{H}^{(2)}<0$, then, instead of instability damped oscillations arise with the frequences $\omega_{01}=\sqrt{\alpha_{1} \alpha_{2}} K$ and $\omega_{02}=\sqrt{\tilde{\alpha}_{H}^{(1)} \tilde{\alpha}_{H}^{(2)}} K$, respectively. It is clear that in the considered linear theory the coefficients $\alpha_{1}^{(W)}, \alpha_{2}^{(W)}, \tilde{\alpha}_{H}^{(1)}$, $\tilde{\alpha}_{H}^{(2)}$ depend not on the amplitudes of the fields, but on the rotation parameters $D_{1,2}$, the Rayleigh number $R a$ and the amplitude of the external force $f_{0}$. Now let us analyze the dependence of these coefficients on the dimensionless parameters. For simplicity let us assume that the dimensionless amplitude of the external force is $f_{0}=10$. Use of the given level of the dimensionless force signifies the choice of a certain level of steady background of small-scale and fast oscillations. It is convenient to replace the Cartesian projections $D_{1}$ and $D_{2}$ in the coefficients $\alpha_{1}^{(W)}, \alpha_{2}^{(W)}, \tilde{\alpha}_{H}^{(1)}$, $\tilde{\alpha}_{H}^{(2)}$ by their projections in the spherical coordinate system $(D, \phi, \theta)$. The coordinate surface $D=$ const is a sphere, where $\theta$ is the latitude $\theta \in[0, \pi], \phi$ is the longitude, $\phi \in[0,2 \pi]$ (see Fig. 2).

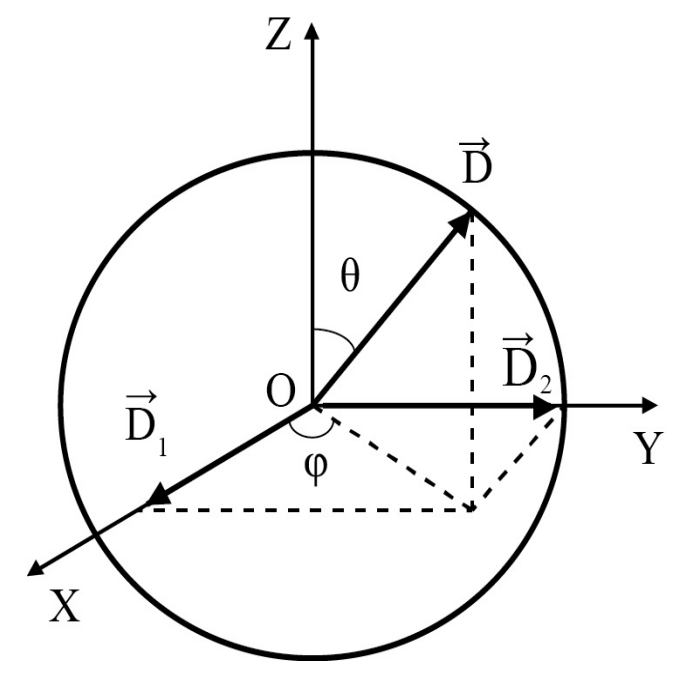

Fig. 2. The case, when the external magnetic field $\bar{B}=0$ shows the relationship of the Cartesian projections of the rotation parameter

$\vec{D}$ (or the angular velocity vector of rotation $\vec{\Omega}$ ) with their projections in a spherical coordinate system.

Let us analyze the dependences of the coefficients $\alpha_{1}, \alpha_{2}, \tilde{\alpha}_{H}^{(1)}, \tilde{\alpha}_{H}^{(2)}$ on the effect of rotation and stratification. For simplicity assume that $D_{1}=D_{2}$, which corresponds to the fixed longitude value $\varphi=\pi / 4+\pi n$, where $n=0,1,2 \ldots k, k$ are integer. In this case the coefficients for vortex and magnetic perturbations are

$$
\begin{gathered}
\alpha=\alpha_{1}^{(W)}=\alpha_{2}^{(W)}=f_{0}^{2} \sqrt{2} D \sin \theta \times \\
\times \frac{4\left(D^{2} \sin ^{2} \theta-2 R a-4\right)(2-R a)+\frac{R a}{2}\left(\left(D^{2} \sin ^{2} \theta-2 R a\right)^{2}+16\right)}{\left(\left(D^{2} \sin ^{2} \theta-2 R a\right)^{2}+16\right)^{2}} \\
\alpha_{H}=\tilde{\alpha}_{H}^{(1)}=\tilde{\alpha}_{H}^{(2)}=\frac{f_{0}^{2} \sqrt{2}}{2} \cdot \frac{D(2+R a) \sin \theta}{\left(D^{2} \sin ^{2} \theta-2 R a\right)^{2}+16}
\end{gathered}
$$

respectively. As can be seen from these relations, at the poles $(\theta=0, \theta=\pi)$ generation of vortex and magnetic perturbations is inefficient, since $\alpha, \alpha_{H} \rightarrow 0$. It means that the large-scale instability occurs in the case when the vector of angular rotation velocity $\vec{\Omega}$ deviates from the axis $Z$. For homogeneous medium $R a=0$ the generation of large-scale vortex and magnetic disturbances is due to the action of an external small-scale non-helical force and the Coriolis force [47]. The coefficient $\alpha$ of vortex perturbations for a rotating stratified electroconductive fluid coincides with the analogous coefficient $\alpha$ for a rotating stratified non-electroconductive fluid obtained in [50]. Therefore, the conclusions of this paper concerning the increase of vortex perturbations may be applied to the problem considered 
here. The dependence of the coefficient $\alpha$ on the parameter of fluid stratification (the Rayleigh number $R a$ ) at the fixed value of latitude $\theta=\pi / 2$ and $D=2.5$ is presented in the left part of Fig. 3 .
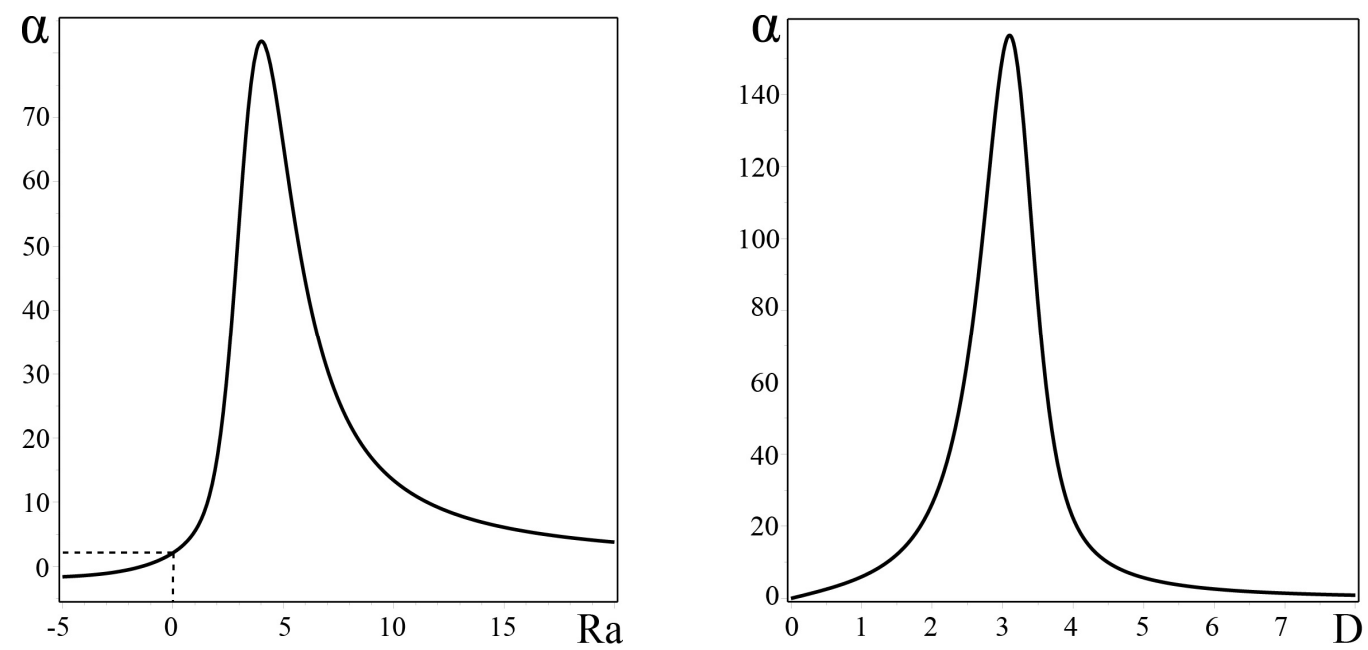

Fig. 3. On the left the plot of $\alpha$ - effect of parameter stratification of the medium $R a$ (Rayleigh number), and on the right the plot of the $\alpha$-effect of the parameter of rotation $D$.

As can be seen, the temperature stratification $(R a \neq 0)$ may increase significantly the coefficient $\alpha$ and, consequently, generate large-scale vortex perturbations faster than in the homogeneous medium. This effect is particularly significant at $R a \rightarrow 5$. When the Rayleigh number increases the value of the coefficient $\alpha$ decreases. Now let us consider the influence of the rotation of the medium on the coefficient $\alpha$. For this purpose, we consider the value of the Rayleigh number as $R a=5$ at $\theta=\pi / 2$. For this case the functional dependence $\alpha(D)$ is presented in the right part of Fig. 3. One can see that at a certain value of the rotation parameter $D$ the coefficient $\alpha$ reaches its maximum $\alpha_{\max }$. With increasing of $D$ the coefficient $\alpha$ smoothly tends to zero, i.e. $\alpha$-effect is suppressed by the rotation of the medium. Now consider the dependence of the coefficient $\alpha_{H}$ on the parameters of stratification and rotation ( $R a$ and $D$, respectively) at the latitude $\theta=\pi / 2$. The dependence of the coefficient $\alpha_{H}$ on the stratification parameter (the Rayleigh number $R a$ ) at $\theta=\pi / 2$ and $D=2.5$ is shown in the left part of Fig. 4.
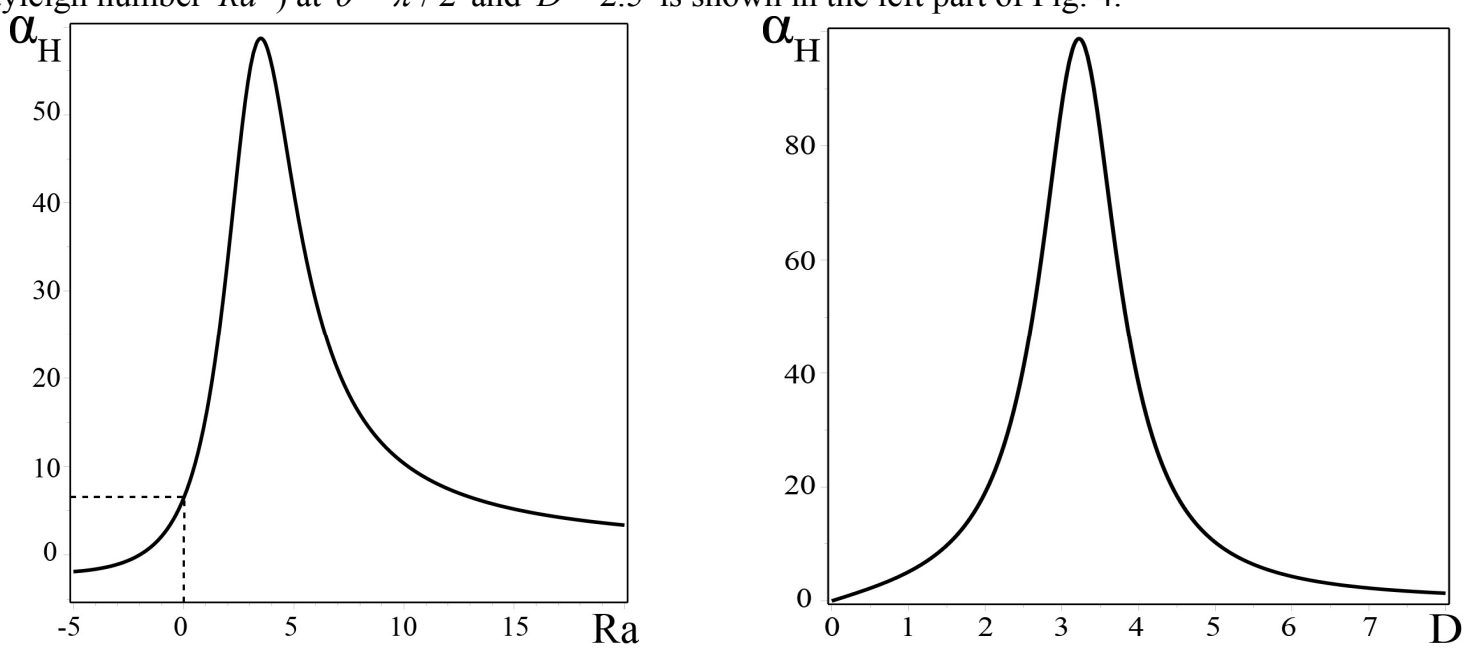

Fig. 4. On the left, the plot of $\alpha_{H}$ - effect of parameter stratification of the medium $R a$ (Rayleigh number), and on the right the plot of the $\alpha_{H}$ - effect of the parameter of rotation $D$.

We can see that the temperature stratification ( $R a \neq 0)$ significantly increases coefficient $\alpha_{H}$, and, consequently, generates the large-scale perturbations faster than in homogeneous medium. Magnetohydrodynamic $\alpha$-effect (or $\alpha_{H}$-effect) also increases at «slow» rotation up to the maximum value $\alpha_{H \max }$. Then with the rise of the parameter $D$ the coefficient $\alpha_{H}$ decreases, but its sign does not change. The analysis of the dependence $\alpha_{H}(D)$ shows that at 
«fast» rotation of the medium MHD $\alpha$-effect is also suppressed (see the right part of Fig. 4). Similar phenomenon, i.e. suppression of $\alpha$-effect by the rotation of turbulent medium is shown in [51].
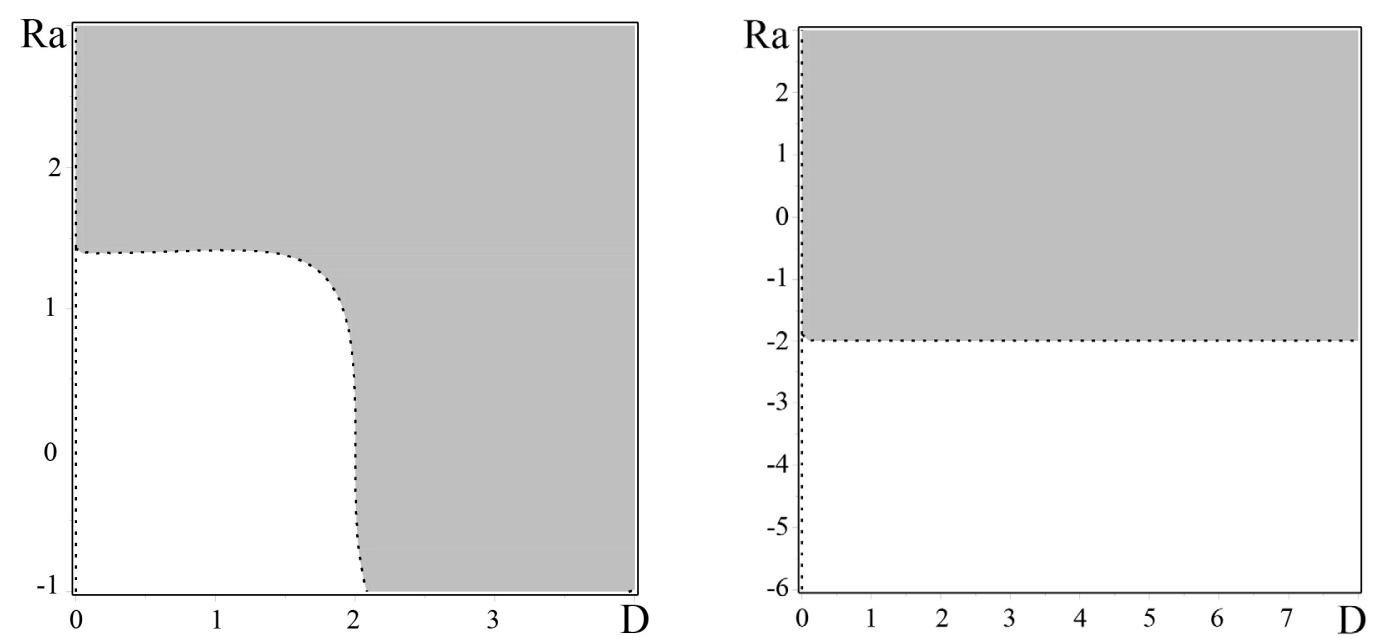

Fig. 5. On the left the plot for $\alpha$ in the plane $(D, R a)$, where the gray color shows the region corresponding to positive values $\alpha>0$ (unstable solutions), and the white shows negative values $\alpha$. On the right is the plot for $\alpha_{H}$ in the plane $(D, R a)$, where the gray color shows the region corresponding to positive values $\alpha_{H}>0$ (unstable solutions), and the white to negative values $\alpha_{H}$.

Fig. 5 shows the plot which represents the influence of rotation and stratification on $\alpha$ and $\alpha_{H}$-effects in the plane $(D, R a)$. Here the regions of instability $\alpha>0, \alpha_{H}>0$ are highlighted in gray. We choose the values of rotation and stratification parameters $D$ and $R a$ for the latitudinal angles $\theta=\pi / 2$ we plot the dependences of the growth rate of the vortex $\Gamma_{1}$ and magnetic $\Gamma_{2}$ perturbations on the wave numbers $K$. These plots have the typical form of $\alpha$-effect (see Fig. 6).
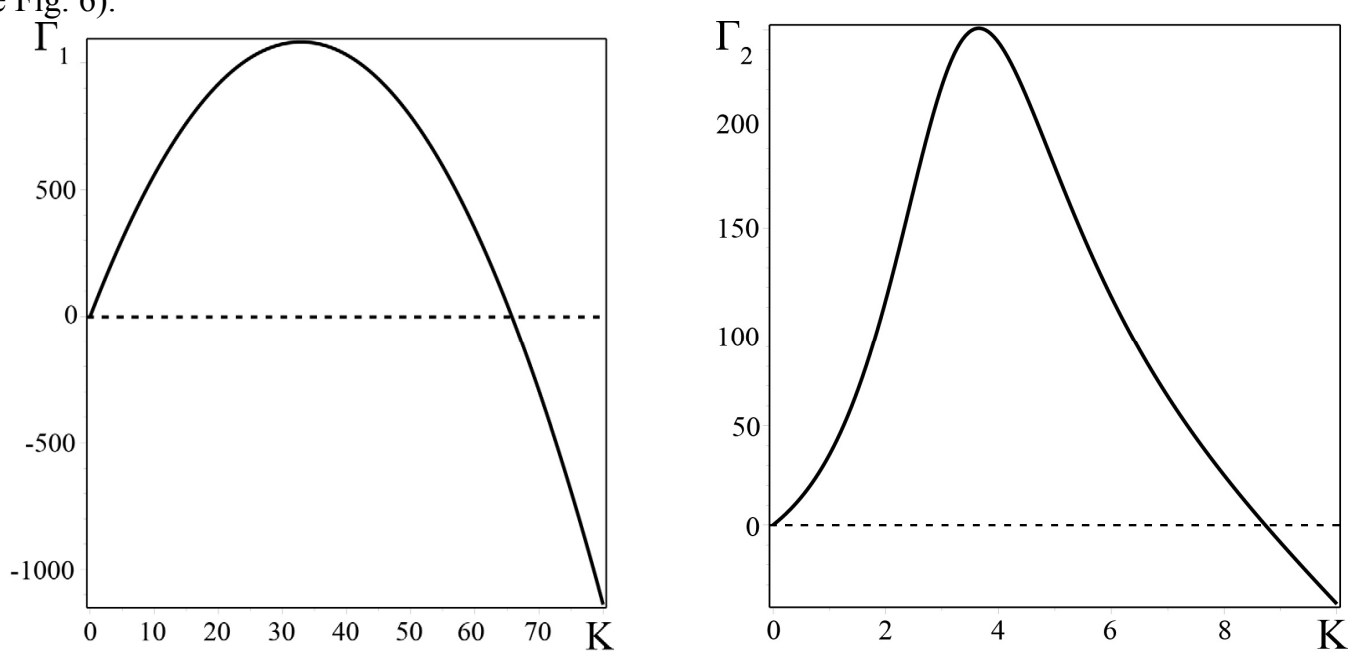

Fig. 6. On the left is the plot of the dependence of the instability increment for $\alpha$-effect on the wave numbers $K$; on the right is a plot of the dependence of the instability increment for the $\alpha$-effect on the wave numbers $K$. The plots are constructed for fixed parameters of stratification $R a=5$ and rotation $D=2.5$.

\section{Analysis of dispersion equation (41) in the presence of the external magnetic field}

Let us study eq. (41) at $\bar{B}_{1,2} \neq 0$. In this case it is transformed into the biquadratic equation :

$$
\left(K^{2}-i \omega\right)^{4}-b\left(K^{2}-i \omega\right)^{2}+a=0,
$$

where

$$
\begin{gathered}
b=K^{2}\left(\alpha_{1}^{(W)} \alpha_{2}^{(W)}+\alpha_{2}^{(H)} \beta_{W}^{(1)}+\tilde{\alpha}_{H}^{(1)} \tilde{\alpha}_{H}^{(2)}+\alpha_{1}^{(H)} \beta_{W}^{(2)}\right)=K^{2} \tilde{b} \\
a=K^{4}\left(\tilde{\alpha}_{H}^{(2)} \alpha_{2}^{(W)}\left(\alpha_{1}^{(W)} \tilde{\alpha}_{H}^{(1)}+\alpha_{1}^{(H)} \beta_{W}^{(1)}\right)+\alpha_{2}^{(H)} \beta_{W}^{(2)}\left(\alpha_{1}^{(H)} \beta_{W}^{(1)}+\tilde{\alpha}_{H}^{(1)} \alpha_{1}^{(W)}\right)\right)=K^{4} \tilde{a}
\end{gathered}
$$


The solution of eq. (47) has the form:

$$
K^{2}-i \omega= \pm K \sqrt{\frac{\tilde{b}}{2} \pm \frac{1}{2} \sqrt{\tilde{b}^{2}-4 \tilde{a}}}
$$

Since we are interested in increasing solutions, it is easy to find the growth rate of large-scale instability from eq. (48):

$$
\Gamma=\operatorname{Im} \omega=\Lambda K-K^{2},
$$

where $\Lambda=\sqrt{\frac{\tilde{b}}{2} \pm \frac{1}{2} \sqrt{\tilde{b}^{2}-4 \tilde{a}}}$ is the coefficient for vortex and magnetic perturbations which has a positive value at $\tilde{b}^{2}>4 \tilde{a}$. The maximum growth rate of instability $\Gamma_{\max }=\Lambda^{2} / 4$ is achieved for the wave numbers $K_{\max }=\Lambda / 2$. In the right part of Fig. 9 is shown the dependence of the growth rate $\Gamma$ of large-scale instability (49) on the wave numbers $K$ for given values of the inclination angle $\theta=\pi / 2$, the amplitude of the extenal force $f_{0}=10$ and the dimensionless parameters $D=2.5, R a=5, Q=10, \bar{B}=0.5$. The form of this plot is typical for $\alpha$-effect (see Fig. 6). As in the previous Section, it is convenient to replace the Cartesian projections $D_{1,2}$ and $\bar{B}_{1,2}$ by their projections in the spherical coordinate system (see Fig. 7).

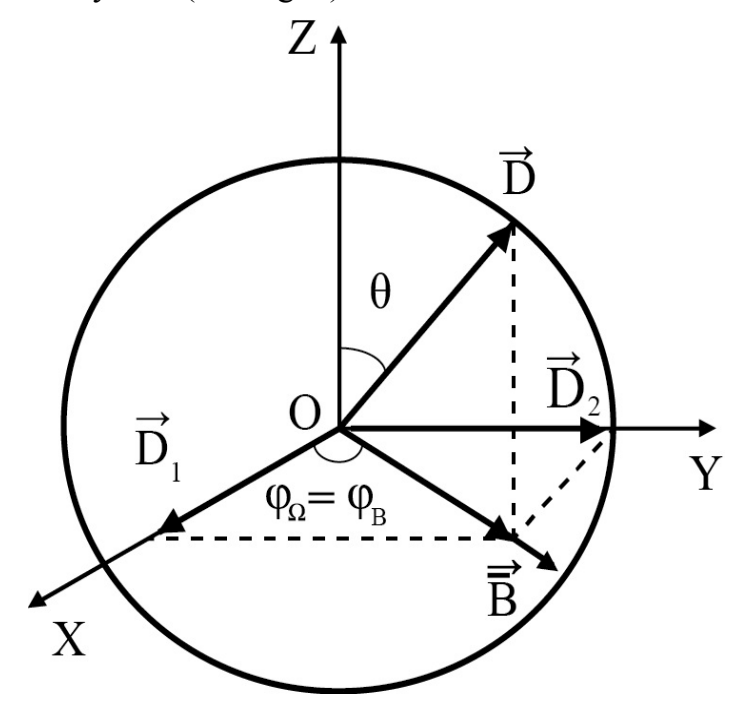

Fig. 7. Here is shown the relationship of the Cartesian projections of the rotation parameter $\vec{D}$ (or the angular velocity vector of rotation $\vec{\Omega}$ ) with their projections in a spherical coordinate system. The direction of the external magnetic field $\bar{B}$ is chosen so that the angles $\varphi$ of deviation from the axis $O X$ for the rotation vector $\varphi_{\Omega}$ and for magnetic field $\varphi_{\Omega}$ and magnetic field $\varphi_{B}$ coincide i.e. $\varphi_{\Omega}=\varphi_{B}$.

Now let us analyze the dependences of the growth rate $\Lambda$ on the effects of rotation $D$, stratification $R a$ and the external magnetic field $\bar{B}$. For simplicity assume that $D_{1}=D_{2}$ and $\bar{B}_{1}=\bar{B}_{2}$, and this corresponds to the fixed value of the angle $\varphi_{\Omega} \approx \varphi_{B}=\pi / 4+\pi n$, where $n=0,1,2 \ldots k, k$ is an integer. In this case the coefficients $\alpha_{1,2}^{(W)}, \alpha_{1,2}^{(H)}, \tilde{\alpha}_{H}^{(1,2)}, \beta_{W}^{(1,2)}$ have the form :

$$
\begin{gathered}
A=\alpha_{1}^{(W)}=\alpha_{2}^{(W)}=f_{0}^{2} \sqrt{2} D \sin \theta \times \\
\times\left[\frac{\left(D^{2} \sin ^{2} \theta-2 R a-4\right)(2-R a)\left(4-Q \bar{B}^{2}\right)}{\left(\left(D^{2} \sin ^{2} \theta-2 R a\right)^{2}+16\right)^{2}}+\frac{R a\left(2-Q \bar{B}^{2}\right)+Q \bar{B}^{2}}{4\left(\left(D^{2} \sin ^{2} \theta-2 R a\right)^{2}+16\right)}\right] \\
B_{H}=\alpha_{1}^{(H)}=\alpha_{2}^{(H)}=\frac{f_{0}^{2}}{8} D Q \bar{B} \sin \theta \times
\end{gathered}
$$




$$
\begin{gathered}
\times\left[\frac{4\left(4-Q \bar{B}^{2}\right)(2-R a)\left(2\left(D^{2} \sin ^{2} \theta-2 R a\right)+(R a+1)^{2}+7\right)}{\left(16+\left(D^{2} \sin ^{2} \theta-2 R a\right)^{2}\right)^{2}}+\frac{Q \bar{B}^{2}-4(R a-1)}{16+\left(D^{2} \sin ^{2} \theta-2 R a\right)^{2}}\right] \\
A_{H}=\tilde{\alpha}_{H}^{(1)}=\tilde{\alpha}_{H}^{(2)}=\frac{f_{0}^{2} \sqrt{2}}{2} \cdot \frac{D\left(2+R a-\frac{Q \bar{B}}{2}+\frac{\sqrt{2}}{2}(2+R a) \bar{B}\right) \sin \theta}{\left(D^{2} \sin ^{2} \theta-2 R a\right)^{2}+16}- \\
-f_{0}^{2} \cdot \frac{2 D \bar{B}(2+R a) \sin \theta}{\left(\left(D^{2} \sin ^{2} \theta-2 R a\right)^{2}+16\right)^{2}} \cdot\left(\left(2\left(D^{2} \sin ^{2} \theta-2 R a\right)+(R a+1)^{2}+7\right)\right. \\
B_{W}=\beta_{W}^{(1)}=\beta_{W}^{(2)}=f_{0}^{2} \cdot \frac{4 D \bar{B}(2+R a) \sin \theta}{\left(\left(D^{2} \sin ^{2} \theta-2 R a\right)^{2}+16\right)^{2}} \cdot\left(D^{2} \sin ^{2} \theta-2 R a-4\right)- \\
-\frac{f_{0}^{2}}{4} \cdot \frac{D \bar{B} R a \sin \theta}{\left(D^{2} \sin ^{2} \theta-2 R a\right)^{2}+16} .
\end{gathered}
$$

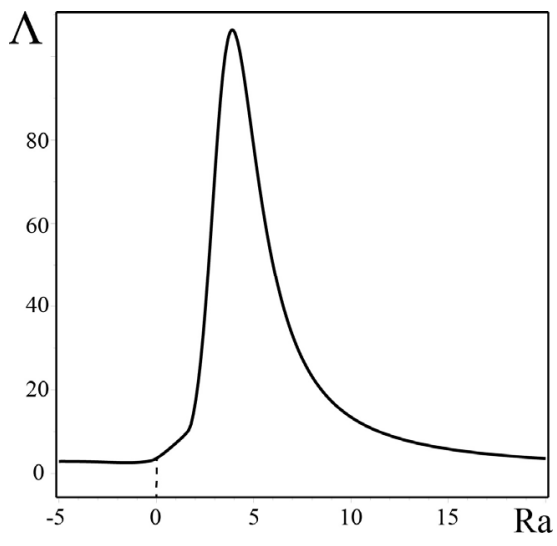

a)

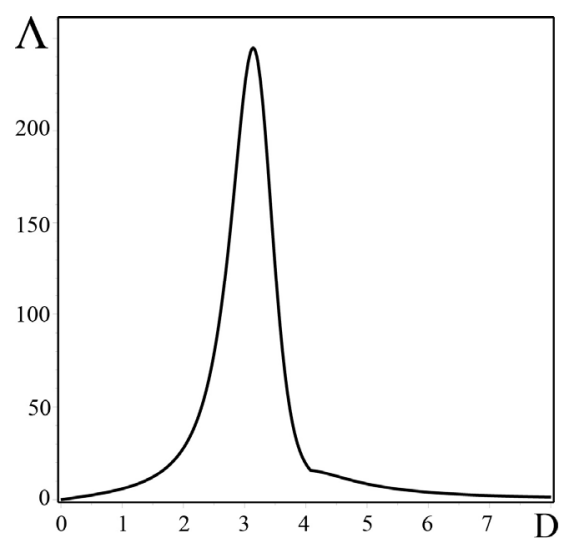

b)

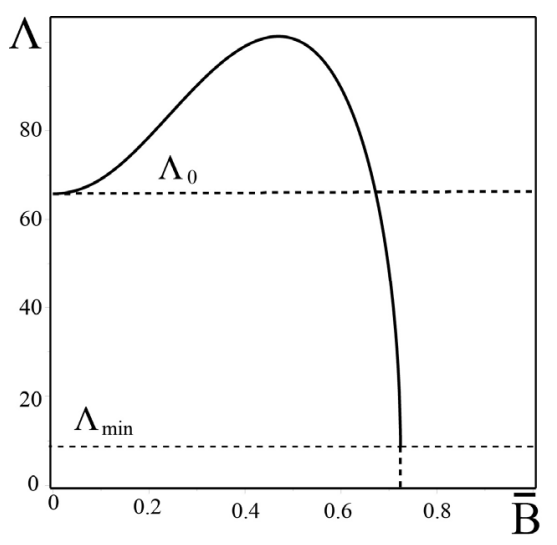

c)

Fig. 8. a) is the plot of the dependence of the $\Lambda$-effect on the stratification parameter of the medium $R a$ (the Rayleigh number); b) is the plot of the dependence of the $\Lambda$-effect on the parameter of rotation of the medium $D$; c) is the plot of the dependence of the $\Lambda$-effect on the external magnetic field $\bar{B}$.

Fig. 8a shows the dependence of the coefficient $\Lambda$ on the Rayleigh number $R a$ at the fixed latitude values $\theta=\pi / 2$ and the dimensionless numbers $D=2.5, Q=10, \bar{B}=0.2$. Assume that the amplitude of the external force $f_{0}=10$. In Fig. 8a the value of the coefficient $\Lambda$ at $R a=0$ (homogeneous medium) are shown by dashed lines. We can see, with the increase of the Rayleigh number $R a \rightarrow 5$ the coefficient $\Lambda$ considerably exceeds its value for a homogeneous medium, i.e. reaches its peak magnitude. Further increase of the parameter $R a$ leads to a drop of the value of $\Lambda$ and, consequently, to less intense generation of the magneto-vortex perturbations. Let us choose the Rayleigh number e.g. on the level of $R a=5$ and find the dependence of the coefficient $\Lambda$ on the rotation parameter $D$ at the external magnetic field $\bar{B}=0.2$ and $Q=10$. The plot presented in Fig. 8b shows the dependence $\Lambda(D)$. Here we observe the increase of $\Lambda$ to a certain maximum value $\Lambda_{\max }$ for $D \approx 3$. With the increase of the parameter $D$ the value of $\Lambda$ diminishes, and generation of magneto-vortex perturbations becomes less efficient. «Fast» rotation of the medium also suppresses the considered $\Lambda$-effect. To clarify the influence of the homogeneous magnetic field $\bar{B}$ on $\Lambda$-effect, let us consider the following parameters: $D=2.5, R a=5, Q=10$. Fig. 8c presents the dependence $\Lambda(\bar{B})$. The upper dashed line indicates the level $\Lambda_{0}$ corresponding to the case when the external magnetic field is absent: $\bar{B}=0$. As seen from this figure, the increase of the magnetic field value gives intensification of the magneto- 
vortex pertubations up to a certain level $\Lambda_{\max } \sim 100$. The lower dashed line in Fig. 8c shows the minimum level of the coefficient $\Lambda_{\min } \approx 9.63$ which corresponds to the value of the magnetic field $\bar{B} \approx 0.72$ for the given parameters $D, R a$ and $Q$. This implies that «strong» external magnetic field suppresses the considered $\Lambda$-effect. For the parameters $D=2.5, R a=5, Q=10$ and $\bar{B}=0.5$ one can find the dependence of the coefficient $\Lambda$ on the angle of deviation $\theta$ for the vector of the angular rotation velocity $\vec{\Omega}$ from the vertical direction $O Z$. This function $\Lambda(\theta)$ is presented in the left part of Fig. 9.
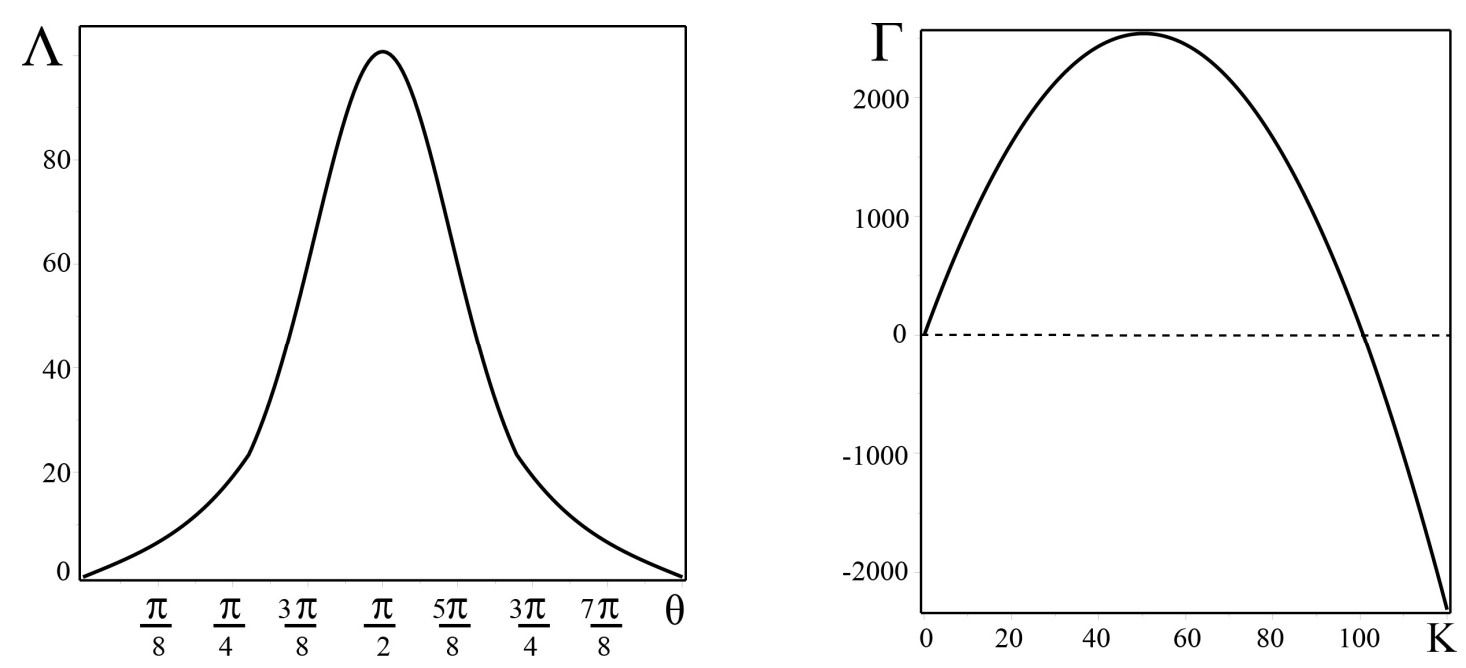

Fig. 9. On the left the plot of the dependence of the $\Lambda$-effect on the angle of inclination $\theta$ of the angular velocity vector $\vec{\Omega}$; on the right the plot of the dependence of the instability increment for the $\Lambda$-effect on the wave numbers $K$.

The generation magneto-vortex perturbations does not occur $(\Lambda \rightarrow 0)$ at $\theta \rightarrow 0$ or $\theta \rightarrow \pi$ (the pole), whereas at $\theta \rightarrow \pi / 2$ (the equator) is more effective.

\section{NONLINEAR STATIONARY STRUCTURES}

When the amplitude of the perturbations $W_{1,2}$ and $H_{1,2}$ increase due to the development of large-scale instability, the linear theory which we consider in the previous Section becomes inapplicable. The evolution of these perturbations is described by the nonlinear system of eqs. (27)-(30). Now study the instability saturation which leads to the formation of nonlinear stationary structures. To describe these structures, let us put $\partial_{T} W_{1}=\partial_{T} W_{2}=\partial_{T} H_{1}=\partial_{T} H_{2}=0$ in the system of eqs. (27)-(30), and then integrate these equations over to $Z$ :

$$
\begin{gathered}
\frac{d \tilde{W}_{1}}{d Z}=-\frac{f_{0}^{2} \sqrt{2} D q_{2} Q_{2}}{16 \tilde{W}_{2}^{2} q_{2}^{2} \tilde{Q}_{2}^{2}+\left[D^{2}+2\left(1-\tilde{W}_{2}^{2}\right)+2 \mu_{2}\right]^{2}+4 \xi_{2}}+C_{1} \\
\frac{d \tilde{W}_{2}}{d Z}=\frac{f_{0}^{2} \sqrt{2} D q_{1} Q_{1}}{16 \tilde{W}_{1}^{2} q_{1}^{2} \tilde{Q}_{1}^{2}+\left[D^{2}+2\left(1-\tilde{W}_{1}^{2}\right)+2 \mu_{1}\right]^{2}+4 \xi_{1}}+C_{2} \\
\frac{d H_{1}}{d Z}=\frac{f_{0}^{2} \sqrt{2} D \tilde{W}_{2} \tilde{Q}_{2}\left(2 H_{2}+\bar{B} \sqrt{2}\right)}{\left(1+\tilde{W}_{2}^{2}\right)\left[16 \tilde{W}_{2}^{2} q_{2}^{2} \tilde{Q}_{2}^{2}+\left[D^{2}+2\left(1-\tilde{W}_{2}^{2}\right)+2 \mu_{2}\right]^{2}+4 \xi_{2}\right]}+C_{3} \\
\frac{d H_{2}}{d Z}=-\frac{f_{0}^{2} \sqrt{2} D \tilde{W}_{1} \tilde{Q}_{1}\left(2 H_{1}+\bar{B} \sqrt{2}\right)}{\left(1+\tilde{W}_{1}^{2}\right)\left[16 \tilde{W}_{1}^{2} q_{1}^{2} \tilde{Q}_{1}^{2}+\left[D^{2}+2\left(1-\tilde{W}_{1}^{2}\right)+2 \mu_{1}\right]^{2}+4 \xi_{1}\right]}+C_{4}
\end{gathered}
$$

Here $\tilde{W}_{1}=1-W_{1}, \tilde{W}_{2}=1-W_{2} ; C_{1}, C_{2}, C_{3}$ and $C_{4}$ are arbitrary integration constants. In order to obtain eqs. 
(54)-(57) we put the Prandtl numbers $\operatorname{Pr}=P m=1$, and substitute the Cartesian projections for $D_{1,2}$ and $\bar{B}_{1,2}$ in the coefficients $\alpha_{(1,2)}, \alpha_{H}^{(1,2)}$ by their projections in the spherical coordinate system (see Fig. 7). For simplicity we use the values of the angles $\varphi_{\Omega}=\varphi_{B}=\pi / 4$ and $\theta=\pi / 2$. In this case, the expressions for $q_{1,2}, Q_{1,2}, \tilde{Q}_{1,2}, \mu_{1,2}, \sigma_{1,2}$, $\chi_{1,2}, \xi_{1,2}$ are also simplified:

$$
\begin{aligned}
& q_{1,2}=1+\frac{Q H_{1,2}\left(2 H_{1,2}+\bar{B} \sqrt{2}\right)}{2\left(1+\tilde{W}_{1,2}^{2}\right)}-\frac{R a}{1+\tilde{W}_{1,2}^{2}}, \quad Q_{1,2}=1-\frac{Q\left(2 H_{1,2}+\bar{B} \sqrt{2}\right)^{2}}{4\left(1+\tilde{W}_{1,2}^{2}\right)}, \\
& \mu_{1,2}=Q H_{1,2}\left(2 H_{1,2}+\bar{B} \sqrt{2}\right)+Q^{2} H_{1,2}^{2}\left(2 H_{1,2}+\bar{B} \sqrt{2}\right)^{2} \cdot \frac{1-\tilde{W}_{1,2}^{2}}{4\left(1+\tilde{W}_{1,2}^{2}\right)}- \\
& -R a \cdot \frac{1+\tilde{W}_{1,2}^{2}+Q H_{1,2}\left(2 H_{1,2}+\bar{B} \sqrt{2}\right) \cdot \frac{1-\tilde{W}_{1,2}^{2}}{1+\tilde{W}_{1,2}^{2}}}{1+\tilde{W}_{1,2}^{2}} \\
& \xi_{1,2}=2 \Xi_{1,2}+2 \tilde{W}_{1,2}^{2} \Pi_{1,2}-2 \tilde{W}_{1,2}^{2}\left(1-\tilde{Q}_{1,2}^{2}\right) \Pi_{1,2}-2\left(1-q_{1,2}^{2}\right) \Xi_{1,2}+\Xi_{1,2} \Pi_{1,2}+\chi_{1,2} \tilde{W}_{1,2}^{2}+\chi_{1,2}\left(1+\sigma_{1,2}\right), \\
& \Xi_{1,2}=-\frac{4 \tilde{W}_{1,2}^{2} \tilde{Q}_{1,2} R a}{1+\tilde{W}_{1,2}^{2}}+\frac{2 \tilde{W}_{1,2}^{2} R a^{2}}{\left(1+\tilde{W}_{1,2}^{2}\right)^{2}}+R a \cdot \frac{1+\tilde{W}_{1,2}^{2}+Q H_{1,2}\left(2 H_{1,2}+\bar{B} \sqrt{2}\right) \cdot \frac{1-\tilde{W}_{1,2}^{2}}{1+\tilde{W}_{1,2}^{2}}}{1+\tilde{W}_{1,2}^{2}} \\
& \Pi_{1,2}=\frac{4 q_{1,2} R a}{1+\tilde{W}_{1,2}^{2}}+\frac{2 R a^{2}}{\left(1+\tilde{W}_{1,2}^{2}\right)^{2}}-R a \cdot \frac{1+\tilde{W}_{1,2}^{2}+Q H_{1,2}\left(2 H_{1,2}+\bar{B} \sqrt{2}\right) \cdot \frac{1-\tilde{W}_{1,2}^{2}}{1+\tilde{W}_{1,2}^{2}}}{1+\tilde{W}_{1,2}^{2}} \\
& \sigma_{1,2}=\frac{Q H_{1,2}\left(2 H_{1,2}+\bar{B} \sqrt{2}\right)}{4\left(1+\tilde{W}_{1,2}^{2}\right)} \cdot\left[4\left(1+\tilde{W}_{1,2}^{2}\right)+Q H_{1,2}\left(2 H_{1,2}+\bar{B} \sqrt{2}\right)\right] \text {, } \\
& \chi_{1,2}=\frac{R a}{1+\tilde{W}_{1,2}^{2}} \cdot\left[R a-\left(2\left(1-\tilde{W}_{1,2}^{2}\right)+Q H_{1,2}\left(2 H_{1,2}+\bar{B} \sqrt{2}\right)\right)\right] \\
& \tilde{Q}_{1,2}=1-\frac{Q H_{1,2}\left(2 H_{1,2}+\bar{B} \sqrt{2}\right)}{2\left(1+\tilde{W}_{1,2}^{2}\right)}+\frac{R a}{1+\tilde{W}_{1,2}^{2}} .
\end{aligned}
$$

Eqs. (54)-(57) constitute the nonlinear dynamic system in 4-dimensional phase space in which phase flow divergence is (equal to) zero. Therefore, the system of eqs. (54)-(57) is conservative. The search for the Hamiltonian of this system is a very difficult task, as the integration is complicated by the dependence of the nonlinear coefficients $\alpha_{(1,2)}, \alpha_{H}^{(1,2)}$ on the fields ( $\vec{W}, \vec{H}$ ), that takes it beyond the class of elementary functions. A complete qualitative analysis of this system is extremely complicated because of a high dimensionality of the phase space and large number of the parameters in the system. According to the general ideas, we can expect that this system of conservative equations may contain structures of resonance and non-resonance tori in the phase space and, consequently, chaotic stationary structures of hydrodynamic and magnetic fields. The considered system of nonlinear eqs. (54)-(57) can be studied using the Poincaré cross-section method. 
STATIONARY CHAOTIC STRUCTURES IN THE ABSENCE OF EXTERNAL MAGNETIC FIELD $\bar{B}=0$.

Let us build the Poincare cross-sections using the standard Mathematica programs for the trajectories in the phase space for the case of rotating elctroconductive fluid stratifified with respect to temperature $(R a \neq 0)$ without the external magnetic field $\bar{B}=0$. All the numerical calculations are performed for the following parameters: $f_{0}=10$, $D=2, Q=1, R a=0.1$ and the constants $C_{1}=1, C_{2}=-1, C_{3}=-0.5, C_{4}=0.5$. For the initial conditions $W_{1}(0)=1.25, \tilde{W}_{2}(0)=1.25, H_{1}(0)=1.4, H_{2}(0)=1.4$ the Poincaré cross-sections presented in Fig. 10a-10b demonstrate regular trajectories for the velocity and magnetic fields. With the increase of the initial perturbation velocity $\tilde{W}_{1}(0)=1.398, \tilde{W}_{2}(0)=1.398, H_{1}(0)=1.4, H_{2}(0)=1.4$ the regular trajectories become chaotic. They correspond to the Poincaré cross-sections shown in Fig. 10c-10d.

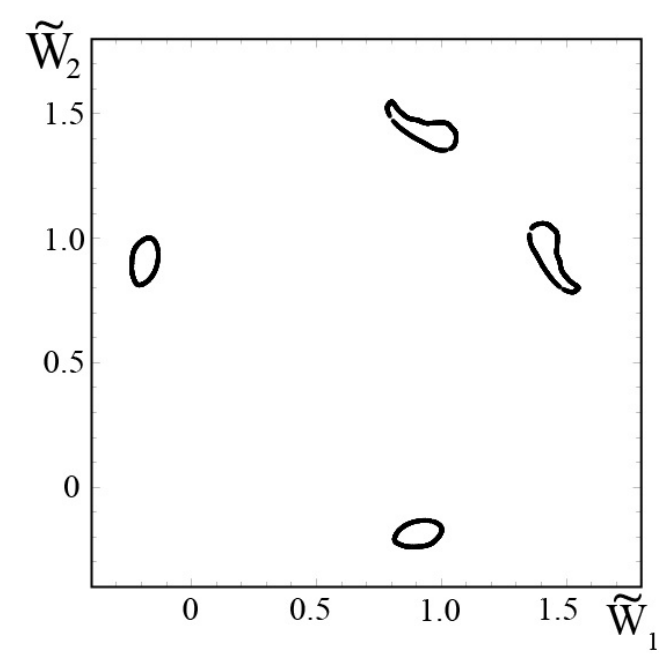

a)

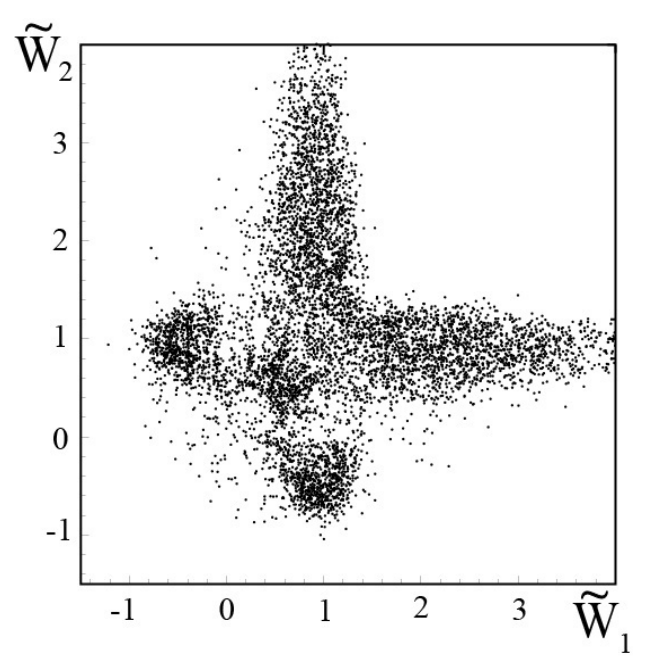

c)

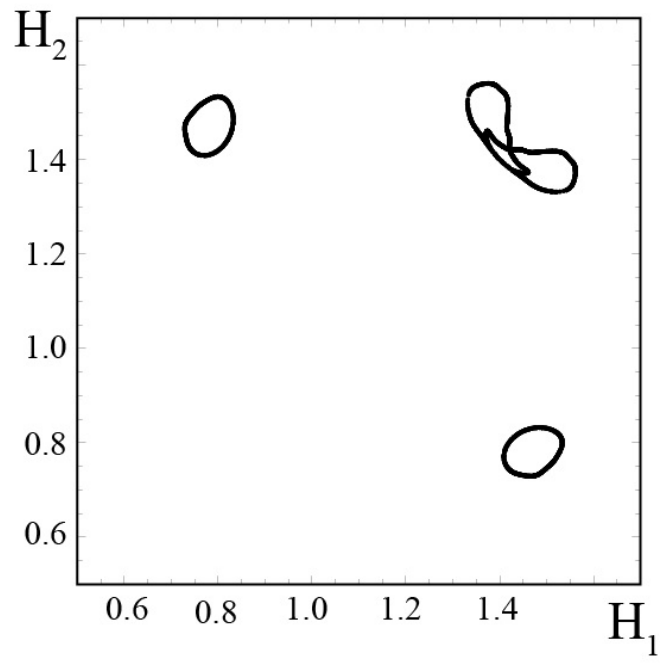

b)

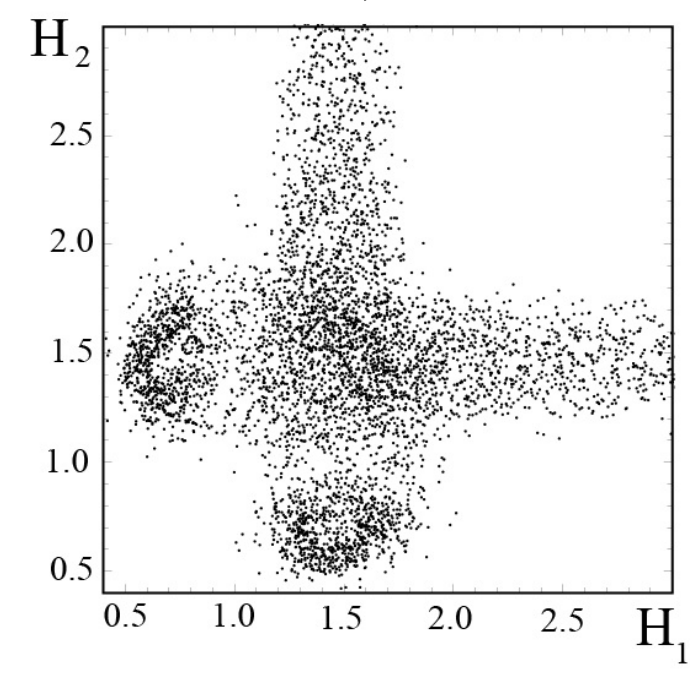

d)

Fig. 10. In the figures a) and b) are shown the Poincare sections for a trajectory with initial conditions $\tilde{W}_{1}(0)=1.25, \tilde{W}_{2}(0)=1.25$, $H_{1}(0)=1.4, H_{2}(0)=1.4$. These are trajectories of regular type, which are wound on the tori. The figures c) and d) correspond to Poincaré sections for a trajectory with initial conditions $\tilde{W}_{1}(0)=1.398, \tilde{W}_{2}(0)=1.398, H_{1}(0)=1.4, H_{2}(0)=1.4$. These pictures show stochastic layers, to which belong the corresponding chaotic trajectories. The calculations were carried out for the case $\bar{B}=0$.

Fig. 11a-11d present the dependence of the stationary large-scale fields on the altitude $Z$. It was obtained numerically for the initial conditions corresponding to the Poincaré cross-sections presented in Fig. 10a-10d. These figures show also the emergence of stationary chaotic solutions for magnetic and vortex fields. To prove the existence of chaotic regime of stationary large-scale fields, we use also the method of autocorrelated function. As is known (see 
e.g. [52]), the autocorrelated function $K(\tau)$ is the value which characterizes the intensity of chaos. It is defined as averaging of the product of random functions $P(t)$ and $P(t+\tau)$ at the moment of time $t$ and $t+\tau$, respectively, over «large» interval of time $\Delta t: K(\tau)=\lim _{\Delta t \rightarrow \infty} \frac{1}{\Delta t} \int_{0}^{\Delta t} P(t) P(t+\tau) d t$. So we consider the coordinate $Z$ as the time $t$, whereas the product $P(t) P(t+\tau)$ consists of 16 components:

$$
P(t) P(t+\tau)=\left[\begin{array}{c}
\tilde{W}_{1}(t) \\
\tilde{W}_{2}(t) \\
H_{1}(t) \\
H_{2}(t)
\end{array}\right]\left[\begin{array}{llll}
\tilde{W}_{1}(t+\tau) & \tilde{W}_{2}(t+\tau) & H_{1}(t+\tau) & H_{2}(t+\tau)
\end{array}\right]
$$

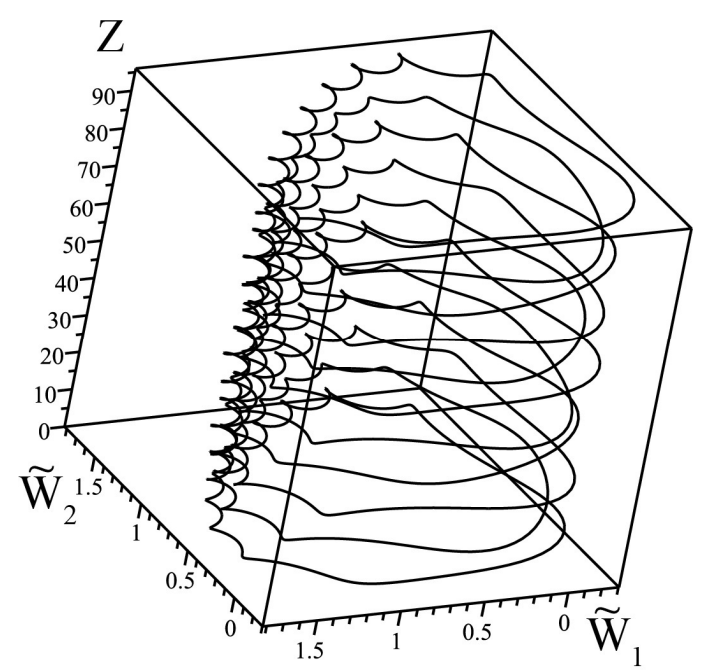

a)

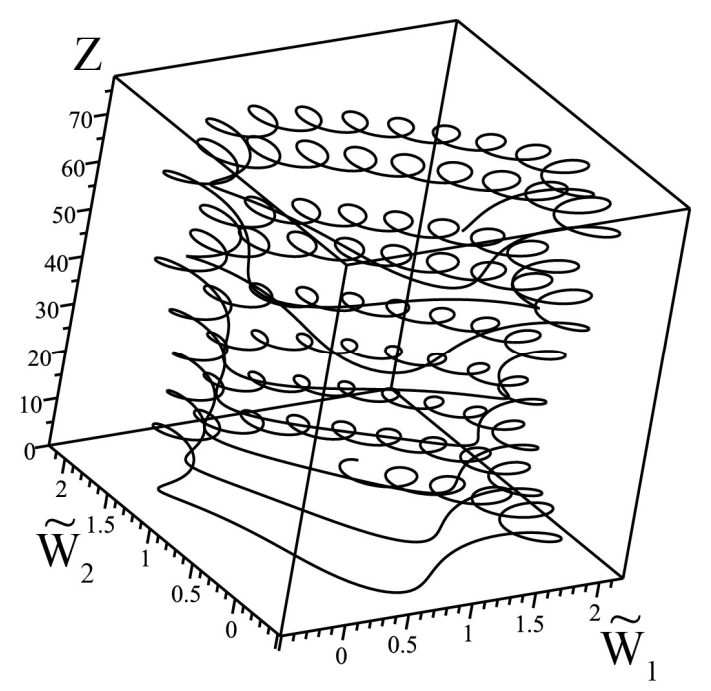

c)

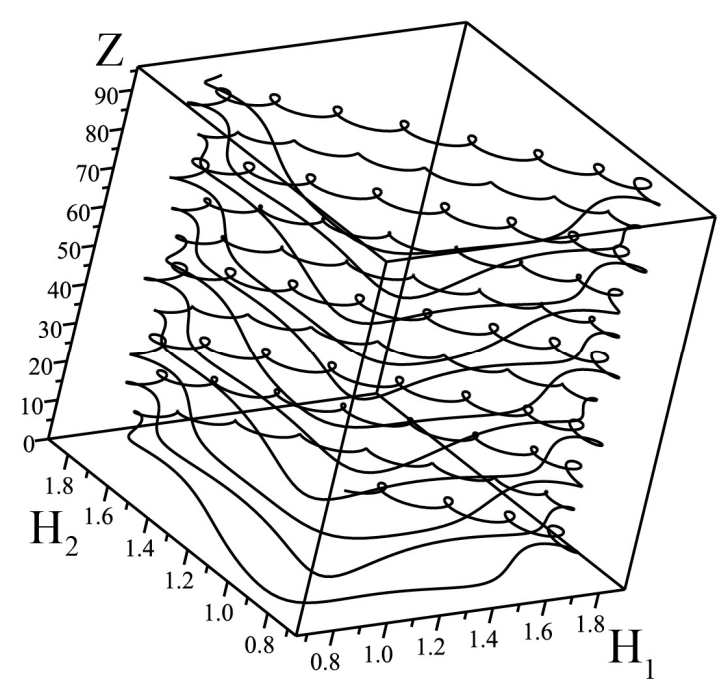

b)

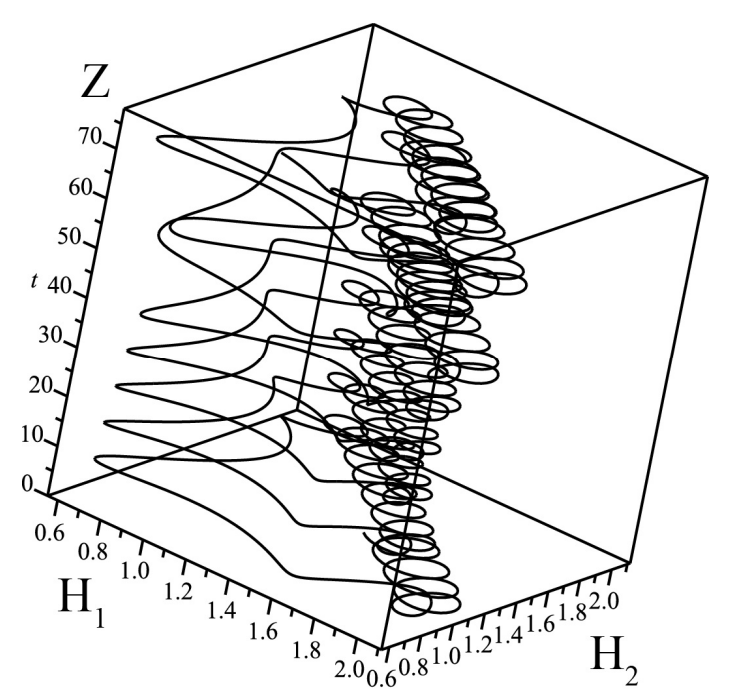

d)

Fig. 11. The upper plots (a), b)) show the dependence of the velocity and magnetic field on the height $Z$ for the numerical solution of equations (54)-(57) with the initial conditions $\tilde{W}_{1}(0)=1.25, \tilde{W}_{2}(0)=1.25, H_{1}(0)=1.4, H_{2}(0)=1.4$. This dependence corresponds to regular motions of the Poincaré section which are shown in Fig. 10a-10b. Plots (c),d)) show the similar dependence for the numerical solution of equations (54)-(57) with the initial conditions: $\tilde{W}_{1}(0)=1.398, \tilde{W}_{2}(0)=1.398$,

$H_{1}(0)=1.4, H_{2}(0)=1.4$. This chaotic dependence corresponds to the Poincaré sections in Fig. 10c-10d. 


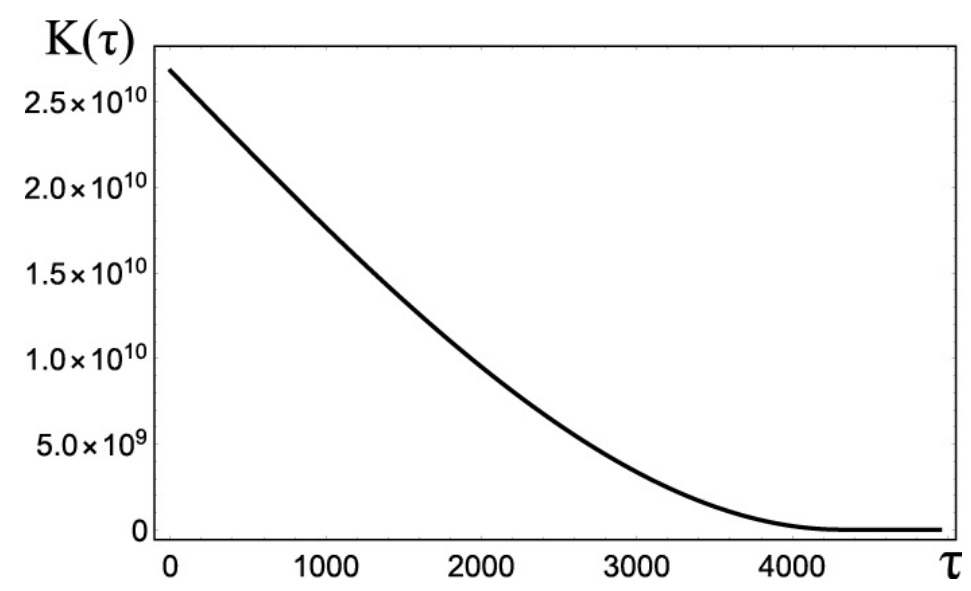

Fig. 12. The plot of the dependence of the autocorrelation function $K_{\tilde{W}_{1} \tilde{W}_{1}}$ on time $\tau$ for a trajectory with initial conditions $\tilde{W}_{1}(0)=1.398, \tilde{W}_{2}(0)=1.398, H_{1}(0)=1.4, H_{2}(0)=1.4$ (chaotic motion) .

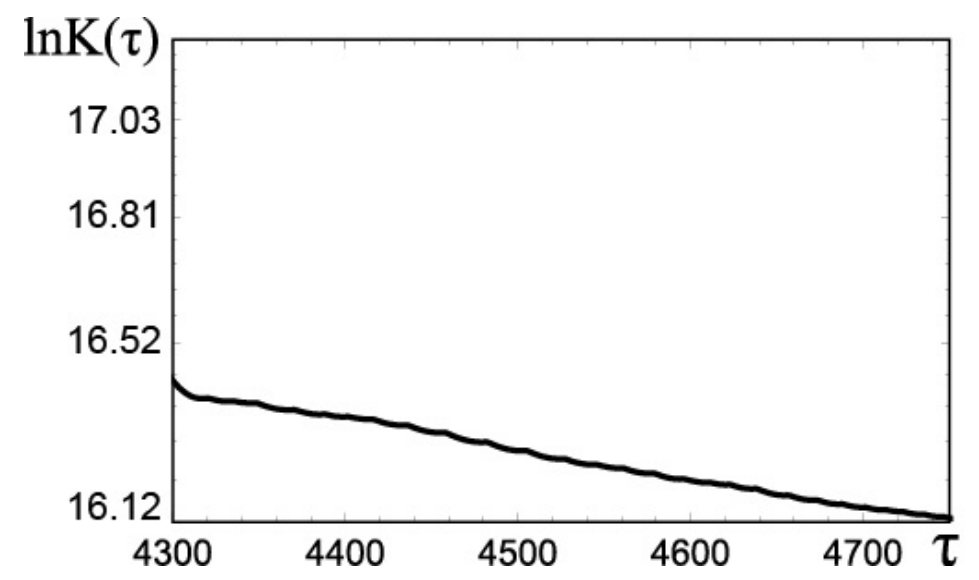

Fig. 13. A rectilinear dependence of the autocorrelation function $K_{\tilde{W}_{1} \tilde{W}_{1}}$ in logarithmic scales from the time interval $\tau$ for strongly chaotic motion.

The plot of the dependence of the autocorrelated function for the component $K_{\tilde{W}_{1} \tilde{W}_{1}}$ on the time $\tau$ is presented in Fig. 12. The case of chaotic motion corresponds to the section of the trajectory with the exponential decay of the function $K_{\tilde{W}_{1} \tilde{W}_{1}}$. It is evident that in this region, the autocorrelation function $K_{\tilde{W}_{1} \tilde{W}_{1}}$ on a logarithmic scale is approximated as a straight line (see Fig. 13). The data presented in Fig. 13 allow to determine the characteristic correlation time $\tau_{c o r} \approx 1324$ of the stationary random process $P_{\tilde{W}_{1}}$. If we take into account the introduced definition of «time» $t$, it becomes clear that we have found the estimated value of the altitude $Z_{\text {cor }} \approx 1324$ corresponding to onset of chaotic motion of the large-scale fields. In Fig. 11c-11в are shown the chaotic solutions for the velocity and magnetic fields of $Z \approx 90$ height which is much less than $Z_{c o r}$. However, even in this case one can see the start of the complex intricate trajectory for the large-scale fields with the increase of the altitude $Z$. Therefore, such trajectories cannot be plotted. Thus, with the increase of the altitude $Z$ up to critical value $Z_{c o r}$, the quasi-periodic motion of the stationary large-scale fields becomes chaotic.

STATIONARY CHAOTIC STRUCTURES IN THE PRESENCE OF EXTERNAL MAGNETIC FIELD $\bar{B} \neq 0$.

Using the standard Matematica programs we construct the Poincaré cross-sections of trajectories in the phase space for the nonlinear system of eqs. (54)-(57) with the external homogeneous magnetic field. All the numerical calculations are carried out for the following dimensionless parameters: $f_{0}=10, D=2, Q=1, R a=0.1$, $\bar{B}=0.1$ and the constants $C_{1}=1, C_{2}=-1, C_{3}=-0.5, C_{4}=0.5$. In Fig. 14a-14b are shown regular trajectories of 
the velocity and magnetic fields built at the numerical solutions of eqs. (54)-(57) with the following initial conditions : $\tilde{W}_{1}(0)=1.31, \tilde{W}_{2}(0)=1.31, H_{1}(0)=1.4, H_{2}(0)=1.4$. These trajectories correspond to quasi-periodic character of motion for large-scale perturbations of the velocity $\left(\tilde{W}_{1,2}\right)$ and magnetic fields $\left(H_{1,2}\right)$. By increasing only the amplitudes of the initial values of perturbations for the magnetic field $\tilde{W}_{1}(0)=1.31, \tilde{W}_{2}(0)=1.31$, $H_{1}(0)=1.8, H_{2}(0)=1.8$ we find that the quasi-periodic motion transforms into chaotic. This case demonstrates the Poincaré cross-sections shown in Fig. 14c-14d.

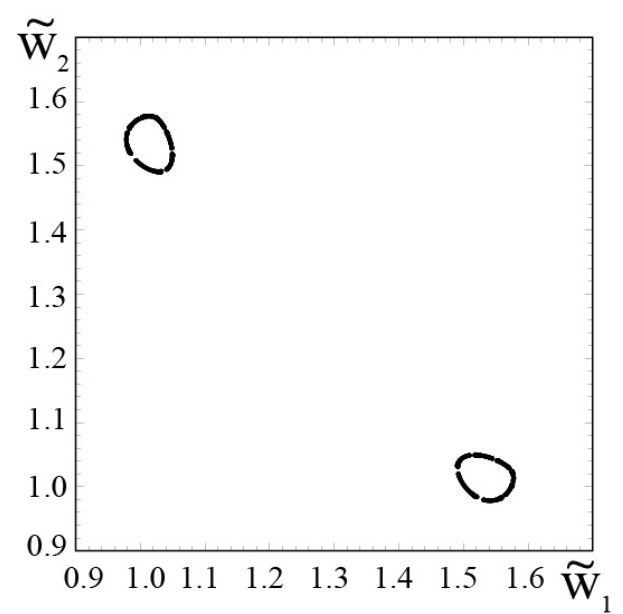

a)

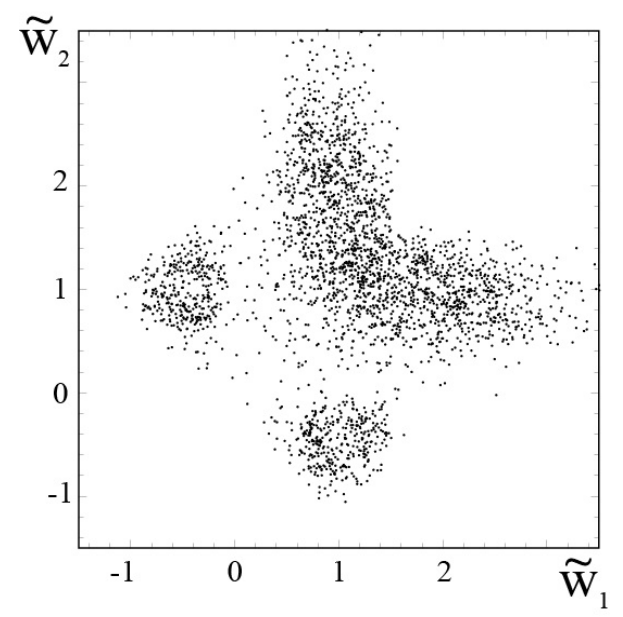

c)

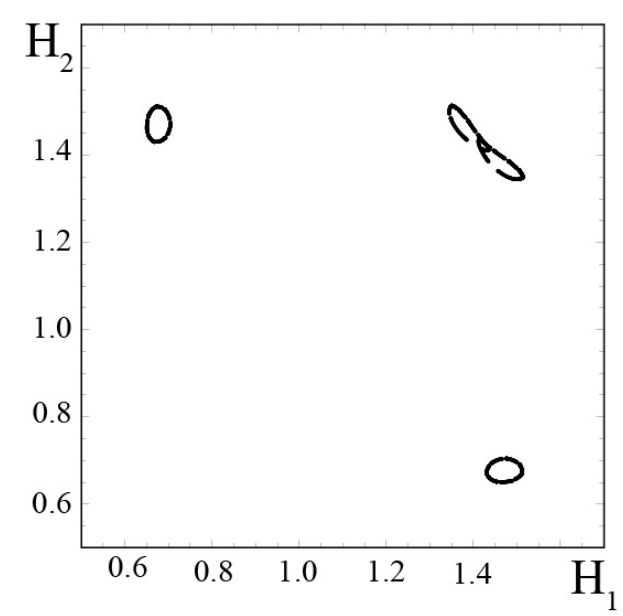

b)

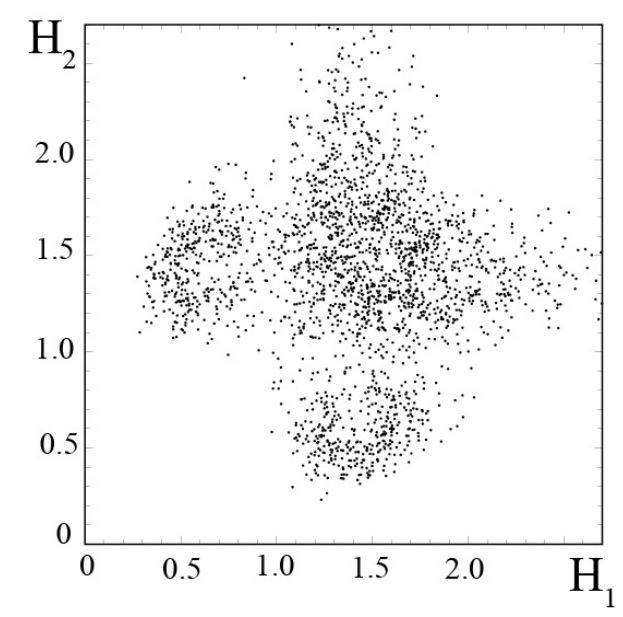

d)

Fig. 14. The figures a) and b) shown the Poincaré sections for a trajectory with initial conditions $\tilde{W}_{1}(0)=1.31, \tilde{W}_{2}(0)=1.31$, $H_{1}(0)=1.4, H_{2}(0)=1.4$. This is a regular type of trajectory, which is wound on the tori. The figures c) and d) correspond to Poincaré sections for a trajectory with initial conditions $\tilde{W}_{1}(0)=1.31, \tilde{W}_{2}(0)=1.31, H_{1}(0)=1.8, H_{2}(0)=1.8$. These pictures show stochastic layers, to which belongs the corresponding chaotic trajectory. The calculations were carried out for the case $\bar{B}=0.1$.

Using the initial data for the regular $\left(\tilde{W}_{1}(0)=1.31, \tilde{W}_{2}(0)=1.31, H_{1}(0)=1.4, H_{2}(0)=1.4\right)$ and chaotic $\left(\tilde{W}_{1}(0)=1.31, \tilde{W}_{2}(0)=1.31, H_{1}(0)=1.8, H_{2}(0)=1.8\right)$ trajectories, we can build numerically the dependence of the stationary large-scale fields on the altitude $Z$ (see Fig. 15a-15d). The emergence of stationary chaotic solutions for the magnetic and vortex fields is also shown in Fig. 15c-15d. To confirm the onset of chaotic regime of the stationary large-scale fields we plot the dependence of the autocorrelated function for the component $K_{\tilde{\tilde{W}}_{1} \tilde{W}_{1}}$ on time $\tau$ (see Fig. 16). The trajectories of chaotic motion correspond to the part of the plot with the exponential decay of function $K_{\tilde{W}_{1} \tilde{W}_{1}}$ in Fig. 16. In the logarithmic scale of the autocorrelated function $K_{\tilde{W}_{1} \tilde{W}_{1}}$ this part is approximated by a straight line (see Fig. 17). Using this plot it is easy to find the estimated value of the characteristic correlation time for a stationary random process: $\tau_{c o r} \approx 2000$. The obtained value of the correlation time corresponds to the altitude 
$Z_{c o r} \approx 2000$. Above this value arise strongly chaotic stationary structures of the large-scale fields. In Fig. 15c-15d are shown chaotic solutions for the velocity and magnetic fields at the altitude $Z \approx 50$ which is significantly less than $Z_{c o r}$. It is evident that for $Z$ tending to a critical value $Z_{c o r}$ the motion trajectories become more intricate and, finally, completely chaotic.

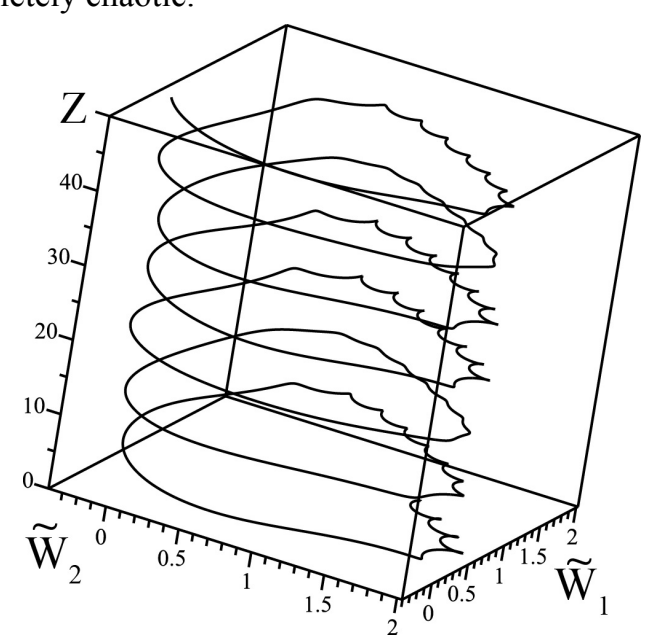

a)

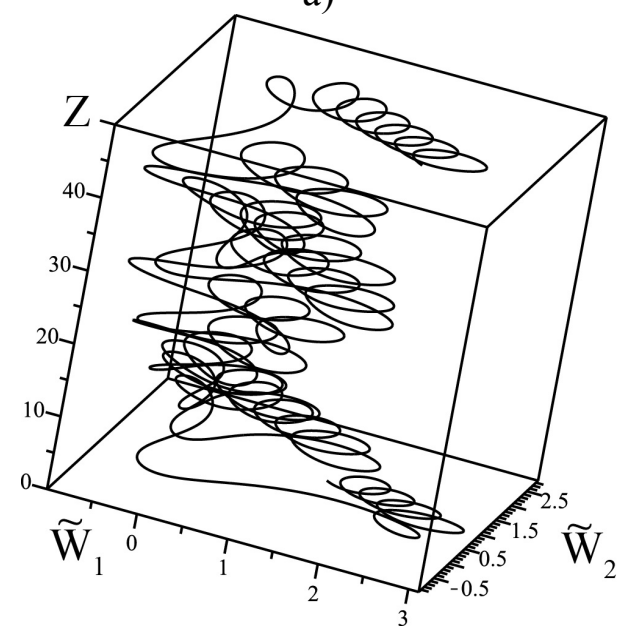

c)

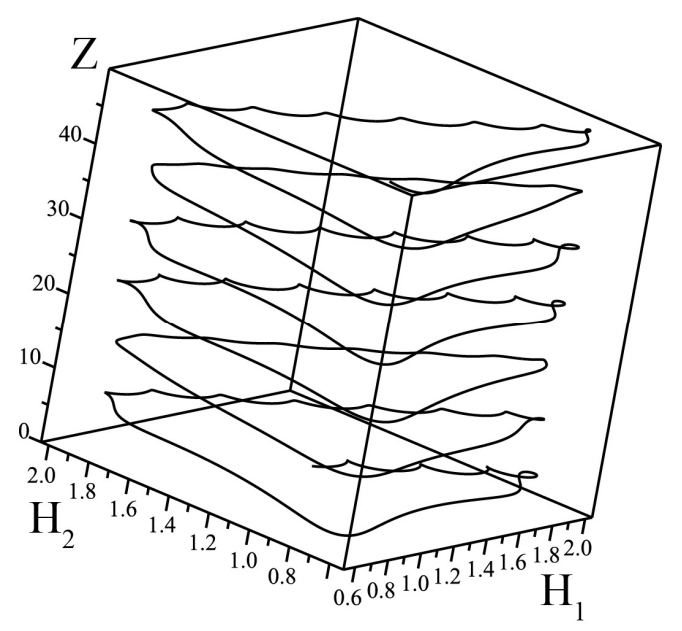

b)

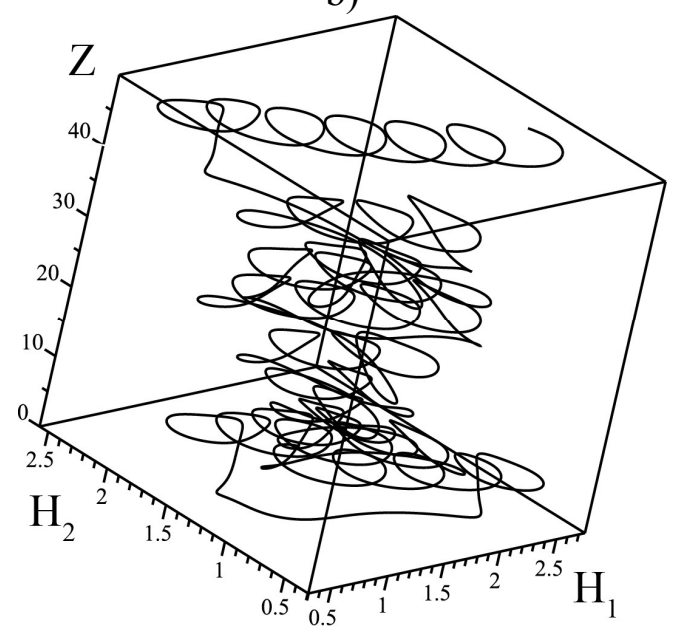

d)

Fig. 15. The upper part (a),b)) shows the dependence of the velocity and magnetic field on the height $Z$ for the numerical solution of equations (54)-(57) with the initial conditions $\tilde{W}_{1}(0)=1.31, \tilde{W}_{2}(0)=1.31, H_{1}(0)=1.4, H_{2}(0)=1.4$. This dependence corresponds to regular motions of the Poincaré section shown on top of Fig. 14a-14b. Below (c), d)) show a similar dependence for the numerical solution of equations (54)-(57) with the initial conditions: $\tilde{W}_{1}(0)=1.31, \tilde{W}_{2}(0)=1.31, H_{1}(0)=1.8, H_{2}(0)=1.8$. This chaotic dependence corresponds to the Poincaré sections shown at the bottom in Fig. 14c-14d

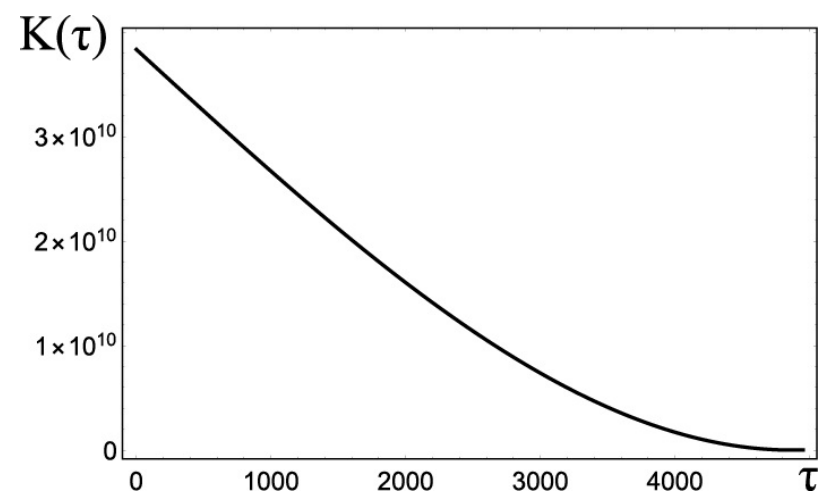

Fig. 16. The plot of the dependence of the autocorrelation function $K_{\tilde{W}_{1} \tilde{W}_{1}}$ on time $\tau$ for a trajectory with initial conditions $\tilde{W}_{1}(0)=1.31, \tilde{W}_{2}(0)=1.31, H_{1}(0)=1.8, H_{2}(0)=1.8$ (chaotic motion) on condition $\bar{B}=0.1$. 


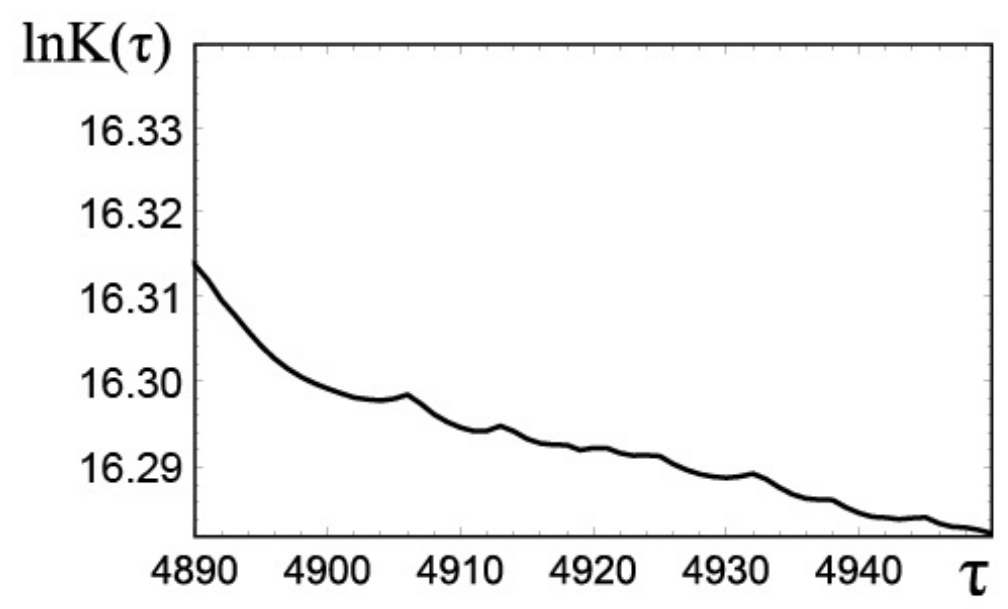

Fig. 17. A rectilinear dependence of the autocorrelation function $K_{\tilde{W}_{1} \tilde{W}_{1}}$ in logarithmic scales from the time interval $\tau$ for strongly chaotic motion on condition $\bar{B}=0.1$.

\section{CONCLUSION}

In this work we have obtained the closed system of nonlinear equations for vortex and magnetic large-scale perturbations (magneto-vortex dynamo) in an obliquely rotating stratified electroconductive fluid in external uniformly magnetic field. At the initial stage small amplitudes of large-scale perturbations increase due to the average helicity $\overrightarrow{\vec{v}_{0} \operatorname{rot}_{0}} \neq 0$ of small-scale motion in a rotating stratified electroconductive fluid excited by the external non-helical force $\vec{F}_{0} \operatorname{rot} \vec{F}_{0}=0$. The mechanism of amplification of the large-scale perturbations is associated with the development of large-scale instability of $\alpha$-effect type. In the absence of external magnetic field $(\overrightarrow{\vec{B}}=0)$ the linear equations of magneto-vortex dynamo are split into two subsystems: vortex and magnetic ones. In this case the largescale vortex and magnetic perturbations are generated du to the development of the instability of HD $\alpha$-effect and MHD $\alpha$-effect, respectively. Both instabilities occur when the vector of angular rotation velocity $\vec{\Omega}$ is deflected from the vertical axis $O Z$. Unlike the case of a homogeneous medium [46-47], the combined effects of rotarion and stratification of the medium (at heating from below) give rise to an essential amplification of the large-scale perturbations. This phenomenon becomes especially noticeable at the parameters of the medium $D \rightarrow 3$ and $R a \rightarrow 5$ (see Fig. 3). In this case arises the mode of the maximal generation of the small-scale helical motion caused by the action of the Coriolis force and temperature inhomogeneity. With the external magnetic field $(\overline{\vec{B}} \neq 0)$ the evolution of the vortex and magnetic perturbations is characterized by a positive feedback due to which the growth rate of the vortex and magnetic large-scale perturbations coincide. The «weak» external magnetic field favours generation of the perturbations, whereas «strong» field suppresses them (see Fig. 8c). Generation of the large-scale vortex and magnetic perturbations also depends on the angle of deflection of the vector of angular rotation velocity $\vec{\Omega}$. It is minimal at $\theta \rightarrow 0$ or $\theta \rightarrow \pi$ (nearby the poles) and maximal at $\theta \rightarrow \pi / 2$ (nearby the equator) (see the left part of Fig. 9). The performed analysis of the influence of rotation on the growth of the vortex and magnetic perturbations shows that at «fast» rotation they are suppressed. With the rise of the perturbation amplitude the instability is stabilized and then becomes stationary. With these conditions arise the nonlinear stationary vortex and magnetic structures. The dynamic system of equations which describes these structures is a Hamiltonian system in a four-dimensional phase space. The possibility of the existence of the large-scale chaotic vortex and magnetic fields in stationary mode is proved by numerical methods. In the absence of external magnetic field $(\vec{B}=0)$ stationary chaotic structures arise in a rotating stratified electroconductive fluid at the increase of the initial velocity of perturbations $\tilde{W}_{1,2}(0)$. With the external magnetic field $(\overline{\vec{B}} \neq 0)$ these structures are formed at the rise of the initial values of the perturbed field $H_{1,2}(0)$.

\section{A. MULTI-SCALE ASYMPTOTIC EXPANSIONS}

APPENDIX

Let us consider the algebraic structure of the asymptotic expansion of eqs.(10)-(13) in different orders in $R$ starting with the lowest of them. In the order $R^{-3}$ we have only one equation: 


$$
\partial_{i} P_{-3}=0 \Rightarrow P_{-3}=P_{-3}(X)
$$

In the order $R^{-2}$ there is the following equation:

$$
\partial_{i} P_{-2}=0 \Rightarrow P_{-2}=P_{-2}(X)
$$

Eqs. (58) and (59) are satisfied automatically, since $P_{-3}$ and $P_{-2}$ are the functions of «slow» variables only. In the order $R^{-1}$ we obtain the system of equations :

$$
\begin{gathered}
\partial_{t} W_{-1}^{i}+W_{-1}^{k} \partial_{k} W_{-1}^{i}=-\partial_{i} P_{-1}-\nabla_{i} P_{-3}+\partial_{k}^{2} W_{-1}^{i}+\varepsilon_{i j k} W_{j} D_{k}+ \\
+\tilde{Q} \varepsilon_{i j k} \varepsilon_{j m l} \partial_{m} B_{-1}^{l} B_{-1}^{k}+\tilde{Q} \varepsilon_{i j k} \varepsilon_{j m l} \partial_{m} B_{-1}^{l} \overline{B_{k}}+e_{i} \tilde{R a} T_{-1} \\
\partial_{t} B_{-1}^{i}-P m^{-1} \partial_{k}^{2} B_{-1}^{i}=\varepsilon_{i j k} \varepsilon_{k n p} \partial_{j} W_{-1}^{n} B_{-1}^{p}+\varepsilon_{i j k} \varepsilon_{k n p} \partial_{j} W_{-1}^{n} \overline{B_{p}} \\
\partial_{t} T_{-1}-P^{-1} \partial_{k}^{2} T_{-1}=-W_{-1}^{k} \partial_{k} T_{-1}+W_{-1}^{z} \\
\partial_{i} W_{-1}^{i}=0, \quad \partial_{i} B_{-1}^{i}=0
\end{gathered}
$$

The averaging of eqs. (60) over the «fast» variables gives the secular equation :

$$
-\nabla_{i} P_{-3}+\varepsilon_{i j k} W_{j} D_{k}+e_{i} \tilde{R a} T_{-1}=0, \quad W_{-1}^{z}=0,
$$

which corresponds to geostrophic equilibrium. In the zero order in $R$ we have the following system of equations:

$$
\begin{gathered}
\partial_{t} v_{0}^{i}+W_{-1}^{k} \partial_{k} v_{0}^{i}+v_{0}^{k} \partial_{k} W_{-1}^{i}=-\partial_{i} P_{0}-\nabla_{i} P_{-2}+\partial_{k}^{2} v_{0}^{i}+ \\
+\varepsilon_{i j k} v_{0}^{j} D_{k}+\tilde{Q} \varepsilon_{i j k} \varepsilon_{j m l}\left(\partial_{m} B_{-1}^{l} B_{0}^{k}+\partial_{m} B_{0}^{l} B_{-1}^{k}\right)+\tilde{Q} \varepsilon_{i j k} \varepsilon_{j m l} \partial_{m} B_{0}^{l} \overline{B_{k}}+e_{i} R a T_{0}+F_{0}^{i} \\
\partial_{t} B_{0}^{i}-P m^{-1} \partial_{k}^{2} B_{0}^{i}=\varepsilon_{i j k} \varepsilon_{k n p}\left(\partial_{j} W_{-1}^{n} B_{0}^{p}+\partial_{j} v_{0}^{n} B_{-1}^{p}\right)+\varepsilon_{i j k} \varepsilon_{k n p} \partial_{j} v_{0}^{n} \overline{B_{p}} \\
\partial_{t} T_{0}-P r^{-1} \partial_{k}^{2} T_{0}=-W_{-1}^{k} \partial_{k} T_{0}-\partial_{k}\left(v_{0}^{k} T_{-1}\right)+v_{0}^{z} \\
\partial_{i} v_{0}^{i}=0, \quad \partial_{i} B_{0}^{i}=0
\end{gathered}
$$

These equations give only one secular term:

$$
\nabla P_{-2}=0 \Rightarrow P_{-2}=\text { const }
$$

Now consider the first-order approximation $R^{1}$ :

$$
\begin{gathered}
\partial_{t} v_{1}^{i}+W_{-1}^{k} \partial_{k} v_{1}^{i}+v_{0}^{k} \partial_{k} v_{0}^{i}+v_{1}^{k} \partial_{k} W_{-1}^{i}+W_{-1}^{k} \nabla_{k} W_{-1}^{i}=-\nabla_{i} P_{-1}-\partial_{i}\left(P_{1}+\bar{P}_{1}\right)+\partial_{k}^{2} v_{1}^{i}+2 \partial_{k} \nabla_{k} W_{-1}^{i}+\varepsilon_{i j k} v_{1}^{j} D_{k}+ \\
+\tilde{Q} \varepsilon_{i j k} \varepsilon_{j m l}\left(\partial_{m} B_{-1}^{l} B_{1}^{k}+\partial_{m} B_{0}^{l} B_{0}^{k}+\partial_{m} B_{1}^{l} B_{-1}^{k}+\nabla_{m} B_{-1}^{l} B_{-1}^{k}\right)+\tilde{Q} \varepsilon_{i j k} \varepsilon_{j m l}\left(\partial_{m} B_{1}^{l} \overline{B_{k}}+\nabla_{m} B_{-1}^{l} \overline{B_{k}}\right)+e_{i} \tilde{R a} T_{1} \\
\partial_{t} B_{1}^{i}-P m^{-1} \partial_{k}^{2} B_{1}^{i}-P m^{-1} 2 \partial_{k} \nabla_{k} B_{-1}^{i}=\varepsilon_{i j k} \varepsilon_{k n p}\left(\partial_{j} W_{-1}^{n} B_{1}^{p}+\partial_{j} v_{0}^{n} B_{0}^{p}+\partial_{j} v_{1}^{n} B_{-1}^{p}+\nabla_{j} W_{-1}^{n} B_{-1}^{p}\right)+ \\
+\varepsilon_{i j k} \varepsilon_{k n p}\left(\partial_{j} v_{1}^{n} \overline{B_{p}}+\nabla_{j} W_{-1}^{n} \overline{B_{p}}\right) \\
\partial_{t} T_{1}-\operatorname{Pr}^{-1} \partial_{k}^{2} T_{1}-\operatorname{Pr}^{-1} 2 \partial_{k} \nabla_{k} T_{-1}=-W_{-1}^{k} \partial_{k} T_{1}-W_{-1}^{k} \nabla_{k} T_{-1}-v_{0}^{k} \partial_{k} T_{0}-v_{1}^{k} \partial_{k} T_{-1}+v_{1}^{z} \\
\partial_{i} v_{1}^{i}+\nabla_{i} W_{-1}^{i}=0, \quad \partial_{i} B_{1}^{i}+\nabla_{i} B_{-1}^{i}=0
\end{gathered}
$$

This system yields the following secular equations:

$$
W_{-1}^{k} \nabla_{k} W_{-1}^{i}=-\nabla_{i} P_{-1}+\tilde{Q} \varepsilon_{i j k} \varepsilon_{j m l}\left(\nabla_{m} B_{-1}^{l} B_{-1}^{k}+\nabla_{m} B_{-1}^{l} \overline{B_{k}}\right)
$$




$$
\begin{gathered}
\varepsilon_{i j k} \varepsilon_{k n p}\left(\nabla_{j} W_{-1}^{n} B_{-1}^{p}+\nabla_{j} W_{-1}^{n} \overline{B_{p}}\right)=0 \\
W_{-1}^{k} \nabla_{k} T_{-1}=0, \quad \nabla_{i} W_{-1}^{i}=0, \quad \nabla_{i} B_{-1}^{i}=0
\end{gathered}
$$

For the second order $R^{2}$ we obtain the equations:

$$
\begin{gathered}
\partial_{t} v_{2}^{i}+W_{-1}^{k} \partial_{k} v_{2}^{i}+v_{0}^{k} \partial_{k} v_{1}^{i}+W_{-1}^{k} \nabla_{k} v_{0}^{i}+v_{0}^{k} \nabla_{k} W_{-1}^{i}+v_{1}^{k} \partial_{k} v_{0}^{i}+v_{2}^{k} \partial_{k} W_{-1}^{i}= \\
=-\nabla_{i} P_{2}-\nabla_{i} P_{0}+\partial_{k}^{2} v_{2}^{i}+2 \partial_{k} \nabla_{k} v_{0}^{i}+\varepsilon_{i j k} v_{2}^{j} D_{k}+ \\
+\tilde{Q} \varepsilon_{i j k} \varepsilon_{j m l}\left(\partial_{m} B_{-1}^{l} B_{2}^{k}+\partial_{m} B_{0}^{l} B_{1}^{k}+\partial_{m} B_{1}^{l} B_{0}^{k}+\partial_{m} B_{2}^{l} B_{-1}^{k}+\nabla_{m} B_{-1}^{l} B_{0}^{k}+\nabla_{m} B_{0}^{l} B_{-1}^{k}\right)+ \\
+\tilde{Q} \varepsilon_{i j k} \varepsilon_{j m l}\left(\partial_{m} B_{2}^{l} \overline{B_{k}}+\nabla_{m} B_{0}^{l} \overline{B_{k}}\right)+e_{i} R a T_{2} \\
\partial_{t} B_{2}^{i}-P m^{-1} \partial_{k}^{2} B_{2}^{i}-P m^{-1} 2 \partial_{k} \nabla_{k} B_{0}^{i}=\varepsilon_{i j k} \varepsilon_{k n p}\left(\partial_{j} W_{-1}^{n} B_{2}^{p}+\partial_{j} v_{0}^{n} B_{1}^{p}+\partial_{j} v_{1}^{n} B_{0}^{p}+\right. \\
=\varepsilon_{i j k} \varepsilon_{k n p}\left(\partial_{j} W_{-1}^{n} B_{2}^{p}+\partial_{j} v_{0}^{n} B_{1}^{p}+\partial_{j} v_{1}^{n} B_{0}^{p}+\partial_{j} v_{2}^{n} B_{-1}^{p}+\nabla_{j} W_{-1}^{n} B_{0}^{p}+\nabla_{j} v_{0}^{n} B_{-1}^{p}\right)+ \\
+\varepsilon_{i j k} \varepsilon_{k n p}\left(\partial_{j} v_{2}^{n} \overline{B_{p}}+\nabla_{j} v_{0}^{n} \overline{B_{p}}\right) \\
\partial_{t} T_{2}-P^{-1} \partial_{k}^{2} T_{2}-P^{-1} 2 \partial_{k} \nabla_{k} T_{0}=-W_{-1}^{k} \partial_{k} T_{2}-W_{-1}^{k} \nabla_{k} T_{0}- \\
-v_{0}^{k} \partial_{k} T_{1}-v_{0}^{k} \nabla_{k} T_{-1}-v_{1}^{k} \partial_{k} T_{0}-v_{2}^{k} \partial_{k} T_{-1}+v_{2}^{z} \\
\partial_{i} v_{2}^{i}+\nabla_{i} v_{0}^{i}=0, \quad \partial_{i} B_{2}^{i}+\nabla_{i} B_{0}^{i}=0
\end{gathered}
$$

As seen after the averaging of the system of eqs. (67) over the «fast» variables, in the order $R^{2}$ secular terms are absent. Finally, let us consider the most significant order $R^{3}$. Here the equations have the following form:

$$
\begin{gathered}
\partial_{t} v_{3}^{i}+\partial_{T} W_{-1}^{i}+W_{-1}^{k} \partial_{k} v_{3}^{i}+v_{0}^{k} \partial_{k} v_{2}^{i}+W_{-1}^{k} \nabla_{k} v_{1}^{i}+v_{0}^{k} \nabla_{k} v_{0}^{i}+v_{1}^{k} \partial_{k} v_{1}^{i}+v_{1}^{k} \nabla_{k} W_{-1}^{i}+ \\
+v_{2}^{k} \partial_{k} W_{-1}^{i}=-\partial_{i} P_{3}-\nabla_{i}\left(P_{1}+\bar{P}_{1}\right)+\partial_{k}^{2} v_{3}^{i}+2 \partial_{k} \nabla_{k} v_{1}^{i}+\Delta W_{-1}^{i}+ \\
+\varepsilon_{i j k} v_{3}^{j} D_{k}+\tilde{Q}_{i j k} \varepsilon_{j m l}\left(\partial_{m} B_{-1}^{l} B_{3}^{k}+\partial_{m} B_{0}^{l} B_{2}^{k}+\partial_{m} B_{1}^{l} B_{1}^{k}+\partial_{m} B_{2}^{l} B_{0}^{k}+\right. \\
\left.+\partial_{m} B_{3}^{l} B_{-1}^{k}++\nabla_{m} B_{-1}^{l} B_{1}^{k}+\nabla_{m} B_{0}^{l} B_{0}^{k}+\nabla_{m} B_{1}^{l} B_{-1}^{k}\right)+ \\
+\tilde{Q} \varepsilon_{i j k} \varepsilon_{j m l}\left(\nabla_{m} B_{3}^{l} \overline{B_{k}}+\nabla_{m} B_{1}^{l} \overline{B_{k}}\right)+e_{i} \operatorname{Ra}_{3} \\
\partial_{t} B_{3}^{i}+\partial_{T} B_{-1}^{i}-P m^{-1} \partial_{k}^{2} B_{3}^{i}-2 P m^{-1} \partial_{k} \nabla_{k} B_{1}^{i}-P m^{-1} \Delta B_{-1}^{i}= \\
\varepsilon_{i j k} \varepsilon_{k n p}\left(\partial_{j} W_{-1}^{n} B_{3}^{p}+\partial_{j} v_{0}^{n} B_{2}^{p}+\partial_{j} v_{1}^{n} B_{1}^{p}+\partial_{j} v_{2}^{n} B_{0}^{p}+\partial_{j} v_{3}^{n} B_{-1}^{p}+\right. \\
\left.+\nabla_{j} W_{-1}^{n} B_{1}^{p}+\nabla_{j} v_{0}^{n} B_{0}^{p}+\nabla_{j} v_{1}^{n} B_{-1}^{p}\right)+\varepsilon_{i j k} \varepsilon_{k n p}\left(\partial_{j} v_{3}^{n} \overline{B_{p}}+\nabla_{j} v_{1}^{n} \overline{B_{p}}\right) \\
\partial_{t} T_{3}+\partial_{T} T_{-1}-\operatorname{Pr}^{-1} \partial_{k}^{2} T_{3}-\operatorname{Pr}^{-1} 2 \partial_{k} \nabla_{k} T_{1}-\operatorname{Pr}^{-1} \Delta T_{-1}= \\
-W_{-1}^{k} \partial_{k} T_{3}-W_{-1}^{k} \nabla_{k} T_{1}-v_{0}^{k} \partial_{k} T_{2}-v_{0}^{k} \nabla_{k} T_{0}- \\
-v_{1}^{k} \nabla_{k} T_{1}-v_{1}^{k} \nabla_{k} T_{-1}-v_{2}^{k} \partial_{k} T_{0}-v_{3}^{k} \partial_{k} T_{-1}+v_{3}^{z} \\
\partial_{i} v_{3}^{i}+\nabla_{i} v_{1}^{i}=0, \quad \partial_{i} B_{3}^{i}+\nabla_{i} B_{1}^{i}=0
\end{gathered}
$$


By averaging this system of equations over the «fast» variables we obtain the basic secular equations which describe the evolution of the large-scale perturbations:

$$
\begin{gathered}
\partial_{T} W_{-1}^{i}-\Delta W_{-1}^{i}+\nabla_{k}\left(\overline{v_{0}^{k} v_{0}^{i}}\right)=-\nabla_{i} \bar{P}_{1}+\tilde{Q} \varepsilon_{i j k} \varepsilon_{j m l} \nabla_{m}\left(\overline{B_{0}^{l} B_{0}^{k}}\right) \\
\partial_{T} B_{-1}^{i}-P m^{-1} \Delta B_{-1}^{i}=\varepsilon_{i j k} \varepsilon_{k n p} \nabla_{j}\left(\overline{v_{0}^{n} B_{0}^{p}}\right) \\
\partial_{T} T_{-1}-P r^{-1} \Delta T_{-1}=-\nabla_{k}\left(\overline{v_{0}^{k} T_{0}}\right)
\end{gathered}
$$

\section{B. SMALL-SCALE FIELDS IN THE ZERO ORDER WITH RESPECT TO $R$}

In Appendix A we obtain the equations of asymptotic expansion in the zero-order approximation. Taking into account the new denotations $\vec{W}=\vec{W}_{-1}, \vec{H}=\vec{B}_{-1}$ we write them down in the following form:

$$
\begin{gathered}
\widehat{D}_{W} v_{0}^{i}=-\partial_{i} P_{0}+\varepsilon_{i j k} v_{0}^{j} D_{k}+\widetilde{Q} H_{k}\left(\partial_{k} B_{0}^{i}-\partial_{i} B_{0}^{k}\right)+\widetilde{Q} \bar{B}_{k}\left(\partial_{k} B_{0}^{i}-\partial_{i} B_{0}^{k}\right)+e_{i} \widetilde{R a} T_{0}+F_{0}^{i} \\
\widehat{D}_{H} B_{0}^{i}=\left(H_{p} \partial_{p}+\bar{B}_{k} \partial_{k}\right) v_{0}^{i} \\
\widehat{D}_{\theta} T_{0}=e_{k} v_{0}^{k} \\
\partial_{i} v_{0}^{i}=\partial_{k} B_{0}^{k}=\partial_{i} F_{0}^{i}=0
\end{gathered}
$$

where the operators are denoted as :

$$
\widehat{D}_{W}=\partial_{t}-\partial_{k}^{2}+W_{k} \partial_{k}, \widehat{D}_{H}=\partial_{t}-P m^{-1} \partial_{k}^{2}+W_{k} \partial_{k}, \widehat{D}_{\theta}=\partial_{t}-P r^{-1} \partial^{2}+W_{k} \partial_{k}
$$

The small-scale oscillations of the magnetic field and the temperature are easily found from Eqs. (73)-(74):

$$
B_{0}^{i}=\frac{\left(H_{p}+\bar{B}_{p}\right) \partial_{p} v_{0}^{i}}{\widehat{D}_{H}}, \quad T_{0}=\frac{v_{0}^{z}}{\widehat{D}_{\theta}}
$$

Now we substitute (76) into (72) and find the pressure $P_{0}$ using the condition of field solenoidality (75) :

$$
P_{0}=\varepsilon_{i j k} \frac{\partial_{i} v_{0}^{j}}{\partial^{2}} D_{k}+e_{i} e_{k} \widetilde{R a} \frac{\partial_{i} v_{0}^{k}}{\partial^{2} \widehat{D}_{\theta}}-\frac{\widetilde{Q}\left(H_{p}+\bar{B}_{p}\right)}{\partial^{2} \widehat{D}_{H}} \partial_{p}\left(H_{k} \partial^{2} v_{0}^{k}\right)
$$

Using the (77) we exclude the pressure from eq. (72) and obtain the equation for the velocity field of the zero-order approximation:

$$
\left[\left(\widehat{D}_{W}-\frac{\widetilde{Q}\left(\left(H_{k} \partial_{k}\right)^{2}+H_{k} \bar{B}_{l} \partial_{k} \partial_{l}\right)}{\widehat{D}_{H}}\right) \delta_{i j}-\left(\widetilde{R a} \frac{e_{j} e_{p}}{\widehat{D}_{\theta}}+\varepsilon_{i j k} D_{k}\right) \widehat{P}_{i p}\right] v_{0}^{j}=F_{0}^{i}
$$

Where $\widehat{P}_{i p}=\delta_{i p}-\frac{\partial_{i} \partial_{p}}{\partial^{2}}$ is the projection operator. In order to find the small-scale field $\vec{v}_{0}$ it is convenient to present eq. (78) in the coordinate form:

$$
\left\{\begin{array}{l}
\hat{d}_{11} v_{0}^{x}+\hat{d}_{12} v_{0}^{y}+\hat{d}_{13} v_{0}^{z}=\widehat{F}_{0}^{x} \\
\hat{d}_{21} v_{0}^{x}+\hat{d}_{22} v_{0}^{y}+\hat{d}_{23} v_{0}^{z}=\widehat{F}_{0}^{y} \\
\hat{d}_{31} v_{0}^{x}+\hat{d}_{32} v_{0}^{y}+\hat{d}_{33} v_{0}^{z}=\widehat{F}_{0}^{z}
\end{array}\right.
$$

The components of the tensor $\hat{d}_{i j}$ have the following form:

$$
\hat{d}_{11}=\widehat{D}_{W}-\frac{\widetilde{Q}\left(\left(H_{k} \partial_{k}\right)^{2}+H_{k} \bar{B}_{l} \partial_{k} \partial_{l}\right)}{\widehat{D}_{H}}+\frac{D_{2} \partial_{x} \partial_{z}-D_{3} \partial_{x} \partial_{y}}{\partial^{2}}, \hat{d}_{12}=\frac{D_{3} \partial_{x}^{2}-D_{1} \partial_{x} \partial_{z}}{\partial^{2}}-D_{3},
$$




$$
\begin{gathered}
\hat{d}_{13}=D_{2}+\frac{D_{1} \partial_{x} \partial_{y}-D_{2} \partial_{x}^{2}}{\partial^{2}}+\widehat{R a} \frac{\partial_{x} \partial_{z}}{\partial^{2} \widehat{D}_{\theta}}, \hat{d}_{21}=D_{3}+\frac{D_{2} \partial_{y} \partial_{z}-D_{3} \partial_{y}^{2}}{\partial^{2}}, \\
\hat{d}_{22}=\widehat{D}_{W}-\frac{\widetilde{Q}\left(\left(H_{k} \partial_{k}\right)^{2}+H_{k} \bar{B} \partial_{k} \partial_{l}\right)}{\widehat{D}_{H}}+\frac{D_{3} \partial_{y} \partial_{x}-D_{1} \partial_{y} \partial_{z}}{\partial^{2}}, \\
\hat{d}_{23}=\widehat{R a} \frac{\partial_{y} \partial_{z}}{\partial^{2} \widehat{D}_{\theta}}+\frac{D_{1} \partial_{y}^{2}-D_{2} \partial_{y} \partial_{x}}{\partial^{2}}-D_{1}, \hat{d}_{31}=\frac{D_{2} \partial_{z}^{2}-D_{3} \partial_{z} \partial_{y}}{\partial^{2}}-D_{2}, \\
\hat{d}_{32}=\frac{D_{3} \partial_{z} \partial_{x}-D_{1} \partial_{z}^{2}}{\partial^{2}}+D_{1}, \hat{d}_{33}=\widehat{D}_{W}-\frac{\widetilde{Q}\left(\left(H_{k} \partial_{k}\right)^{2}+H_{k} \bar{B}_{l} \partial_{k} \partial_{l}\right)}{\widehat{D}_{H}}+ \\
+\frac{D_{1} \partial_{z} \partial_{y}-D_{2} \partial_{z} \partial_{x}}{\partial^{2}}-\frac{\widetilde{R a}}{\widehat{D}_{\theta}}+\widetilde{R a} \frac{\partial_{z}^{2}}{\partial^{2} \widehat{D}_{\theta}} .
\end{gathered}
$$

It is obvious that the solution for the system (79) can be found using the Cramer rule:

$$
\begin{aligned}
& v_{0}^{x}=u_{0}=\frac{1}{\Delta}\left\{\left(\hat{d}_{22} \hat{d}_{33}-\hat{d}_{32} \hat{d}_{23}\right) F_{0}^{x}+\left(\hat{d}_{13} \hat{d}_{32}-\hat{d}_{12} \hat{d}_{33}\right) F_{0}^{y}+\left(\hat{d}_{12} \hat{d}_{23}-\hat{d}_{13} \hat{d}_{22}\right) F_{0}^{z}\right\} \\
& v_{0}^{y}=v_{0}=\frac{1}{\Delta}\left\{\left(\hat{d}_{23} \hat{d}_{31}-\hat{d}_{21} \hat{d}_{33}\right) F_{0}^{x}+\left(\hat{d}_{11} \hat{d}_{33}-\hat{d}_{13} \hat{d}_{31}\right) F_{0}^{y}+\left(\hat{d}_{13} \hat{d}_{21}-\hat{d}_{11} \hat{d}_{23}\right) F_{0}^{z}\right\} \\
& v_{0}^{z}=w_{0}=\frac{1}{\Delta}\left\{\left(\hat{d}_{21} \hat{d}_{32}-\hat{d}_{22} \hat{d}_{31}\right) F_{0}^{x}+\left(\hat{d}_{12} \hat{d}_{31}-\hat{d}_{11} \hat{d}_{32}\right) F_{0}^{y}+\left(\hat{d}_{11} \hat{d}_{22}-\hat{d}_{12} \hat{d}_{21}\right) F_{0}^{z}\right\}
\end{aligned}
$$

Here $\Delta$ is the determinant of the system of equations (79), which in the open form is:

$$
\Delta=\hat{d}_{11} \hat{d}_{22} \hat{d}_{33}+\hat{d}_{21} \hat{d}_{32} \hat{d}_{13}+\hat{d}_{12} \hat{d}_{23} \hat{d}_{31}-\hat{d}_{13} \hat{d}_{22} \hat{d}_{31}-\hat{d}_{32} \hat{d}_{23} \hat{d}_{11}-\hat{d}_{21} \hat{d}_{12} \hat{d}_{33}
$$

Now let us present the external force $\vec{F}_{0}$ in the complex form:

$$
\vec{F}_{0}=\vec{i} \frac{f_{0}}{2} e^{i \phi_{2}}+\vec{j} \frac{f_{0}}{2} e^{i \phi_{1}}+c . c .
$$

Then all the operators contained in (80)-(83) act on the eigenfunctions from the left:

$$
\begin{gathered}
\widehat{D}_{W, H, \theta} e^{i \phi_{1}}=e^{i \phi_{1}} \widehat{D}_{W, H, \theta}\left(\vec{\kappa}_{1},-\omega_{0}\right), \quad \widehat{D}_{W, H, \theta} e^{i \phi_{2}}=e^{i \phi_{2}} \widehat{D}_{W, H, \theta}\left(\vec{\kappa}_{2},-\omega_{0}\right), \\
\Delta e^{i \phi_{1}}=e^{i \phi_{1}} \Delta\left(\vec{\kappa}_{1},-\omega_{0}\right), \quad \Delta e^{i \phi_{2}}=e^{i \phi_{2}} \Delta\left(\vec{\kappa}_{2},-\omega_{0}\right)
\end{gathered}
$$

To simplify the formulae, assume that $\kappa_{0}=1, \omega_{0}=1$ and introduce the new denotations :

$$
\begin{array}{cc}
\widehat{D}_{W}\left(\vec{\kappa}_{1},-\omega_{0}\right)=\widehat{D}_{W_{1}}^{*}=1-i\left(1-W_{1}\right), & \widehat{D}_{W}\left(\vec{\kappa}_{2},-\omega_{0}\right)=\widehat{D}_{W_{2}}^{*}=1-i\left(1-W_{2}\right) \\
\widehat{D}_{H}\left(\vec{\kappa}_{1},-\omega_{0}\right)=\widehat{D}_{H_{1}}^{*}=P m^{-1}-i\left(1-W_{1}\right), & \widehat{D}_{H}\left(\vec{\kappa}_{2},-\omega_{0}\right)=\widehat{D}_{H_{2}}^{*}=P m^{-1}-i\left(1-W_{2}\right) \\
\widehat{D}_{\theta}\left(\vec{\kappa}_{1},-\omega_{0}\right)=\widehat{D}_{\theta_{1}}^{*}=P r^{-1}-i\left(1-W_{1}\right), & \widehat{D}_{\theta}\left(\vec{\kappa}_{2},-\omega_{0}\right)=\widehat{D}_{\theta_{2}}^{*}=P r^{-1}-i\left(1-W_{2}\right)
\end{array}
$$

Note that the complex-conjugate terms are marked by asterisk. Further in the calculations some components in the tensors $\hat{d}_{i j}\left(\vec{\kappa}_{1},-\omega_{0}\right)$ and $\hat{d}_{i j}\left(\vec{\kappa}_{2},-\omega_{0}\right)$ vanish, and there remain the non-zero components:

$$
\hat{d}_{11}\left(\vec{\kappa}_{1},-\omega_{0}\right)=\widehat{D}_{W_{1}}^{*}+\frac{\widetilde{Q} H_{1}\left(H_{1}+\bar{B}_{1}\right)}{\widehat{D}_{H_{1}}^{*}}, \hat{d}_{12}\left(\vec{\kappa}_{1},-\omega_{0}\right)=0, \hat{d}_{13}\left(\vec{\kappa}_{1},-\omega_{0}\right)=0,
$$




$$
\begin{gathered}
\hat{d}_{21}\left(\vec{\kappa}_{1},-\omega_{0}\right)=D_{3}, \hat{d}_{22}\left(\vec{\kappa}_{1},-\omega_{0}\right)=\hat{d}_{11}\left(\vec{\kappa}_{1},-\omega_{0}\right), \hat{d}_{23}\left(\vec{\kappa}_{1},-\omega_{0}\right)=-D_{1}, \\
\hat{d}_{31}\left(\vec{\kappa}_{1},-\omega_{0}\right)=-D_{2}, \hat{d}_{32}\left(\vec{\kappa}_{1},-\omega_{0}\right)=D_{1}, \hat{d}_{33}\left(\vec{\kappa}_{1},-\omega_{0}\right)=\hat{d}_{22}\left(\vec{\kappa}_{1},-\omega_{0}\right)-\frac{\widetilde{R a}}{\widehat{D}_{\theta_{1}}^{*}} \\
\hat{d}_{11}\left(\vec{\kappa}_{2},-\omega_{0}\right)=\widehat{D}_{W_{2}}^{*}+\frac{\widetilde{Q} H_{2}\left(H_{2}+\bar{B}_{2}\right)}{\widehat{D}_{H_{2}}^{*}}, \hat{d}_{12}\left(\vec{\kappa}_{2},-\omega_{0}\right)=-D_{3}, \hat{d}_{13}\left(\vec{\kappa}_{2},-\omega_{0}\right)=D_{2}, \\
\hat{d}_{21}\left(\vec{\kappa}_{2},-\omega_{0}\right)=0, \hat{d}_{22}\left(\vec{\kappa}_{2},-\omega_{0}\right)=\hat{d}_{11}\left(\vec{\kappa}_{2},-\omega_{0}\right), \hat{d}_{23}\left(\vec{\kappa}_{2},-\omega_{0}\right)=0, \\
\hat{d}_{31}\left(\vec{\kappa}_{2},-\omega_{0}\right)=-D_{2}, \hat{d}_{32}\left(\vec{\kappa}_{1},-\omega_{0}\right)=D_{1}, \hat{d}_{33}\left(\vec{\kappa}_{2},-\omega_{0}\right)=\hat{d}_{22}\left(\vec{\kappa}_{2},-\omega_{0}\right)-\frac{\widetilde{R a}}{\widehat{D}_{\theta_{2}}^{*}}
\end{gathered}
$$

Taking into account the expressions (87)-(88) we find the velocity fields in the zero-order approximation:

$$
\begin{gathered}
u_{0}=\frac{f_{0}}{2} \frac{\widehat{B}_{2}^{*}}{\widehat{A}_{2}^{*} \widehat{B}_{2}^{*}+D_{2}^{2}} e^{i \phi_{2}}+\text { c.c. }=u_{03}+u_{04} \\
v_{0}=\frac{f_{0}}{2} \frac{\widehat{B}_{1}}{\hat{A}_{1}^{*} \widehat{B}_{1}^{*}+D_{1}^{2}} e^{i \phi_{1}}+\text { c.c. }=v_{01}+v_{02} \\
w_{0}=-\frac{f_{0}}{2} \frac{D_{1}}{\hat{A}_{1}^{*} \widehat{B}_{1}^{*}+D_{1}^{2}} e^{i \phi_{1}}+\frac{f_{0}}{2} \frac{D_{2}}{\widehat{A}_{2}^{*} \widehat{B}_{2}^{*}+D_{2}^{2}} e^{i \phi_{2}}+\text { c.c. }=w_{01}+w_{02}+w_{03}+w_{04}
\end{gathered}
$$

where

$$
\widehat{A}_{1,2}^{*}=\widehat{D}_{W_{1,2}}^{*}+\frac{\widetilde{Q} H_{1,2}}{\widehat{D}_{H_{1,2}}^{*}}\left(H_{1,2}+\bar{B}_{1,2}\right), \widehat{B}_{1,2}^{*}=\widehat{A}_{1,2}^{*}-\frac{\widetilde{R a}}{\widehat{D}_{\theta_{1,2}}^{*}} .
$$

The velocity components satisfy the following relations: $w_{02}=\left(w_{01}\right)^{*}, w_{04}=\left(w_{03}\right)^{*}, v_{02}=\left(v_{01}\right)^{*}, v_{04}=\left(v_{03}\right)^{*}$, $u_{02}=\left(u_{01}\right)^{*}, u_{04}=\left(u_{03}\right)^{*}$. In the limiting case of non-electroconductive fluid $(\sigma=0)$, without temperature gradient $(\nabla \bar{T}=0)$ and external magnetic field $\left(\bar{B}_{1,2}=0\right)$ the formulae (89)-(91) coincide with the results obtained in [41]. Now we calculate the small-scale oscillations of the magnetic field $\vec{B}_{0}$ using the expressions (76) and (89)-(91):

$$
\begin{gathered}
B_{0}^{x}=\tilde{u}_{0}=\frac{f_{0}}{2} \frac{i\left(H_{2}+\bar{B}_{2}\right) \widehat{B}_{2}^{*}}{\widehat{D}_{H_{2}}^{*}\left(\hat{A}_{2}^{*} \widehat{B}_{2}^{*}+D_{2}^{2}\right)} e^{i \phi_{2}}+c . c .=\tilde{u}_{03}+\tilde{u}_{04} \\
B_{0}^{y}=\tilde{v}_{0}=\frac{f_{0}}{2} \frac{i\left(H_{1}+\bar{B}_{1}\right) \widehat{B}_{1}^{*}}{\widehat{D}_{H_{1}}^{*}\left(\widehat{A}_{1}^{*} \widehat{B}_{1}^{*}+D_{1}^{2}\right)} e^{i \phi_{1}}+c . c .=\tilde{u}_{03}+\tilde{u}_{04} \\
B_{0}^{z}=\tilde{w}_{0}=-\frac{f_{0}}{2} \frac{i\left(H_{1}+\bar{B}_{1}\right) D_{1}}{\widehat{D}_{H_{1}}^{*}\left(\widehat{A}_{1}^{*} \widehat{B}_{1}^{*}+D_{1}^{2}\right)} e^{i \phi_{1}}+\frac{f_{0}}{2} \frac{i\left(H_{2}+\bar{B}_{2}\right) D_{2}}{\widehat{D}_{H_{2}}^{*}\left(\widehat{A}_{2}^{*} \widehat{B}_{2}^{*}+D_{2}^{2}\right)} e^{i \phi_{2}}+c . c .= \\
=w_{01}+w_{02}+w_{03}+w_{04}
\end{gathered}
$$

In the expressions for the small-scale oscillations $\left(\vec{v}_{0}, \vec{B}_{0}, T_{0}\right)$ the component of the angular velocity $D_{3}$ is absent due to the choice of the external force. Further Eqs. (89)-(95) will be used while calculating the correlation functions.

C. CALCULATION OF REYNOLDS AND MAXWELL STRESSES AND TURBULENT E.M.F.

To close the system of eqs. (17)-(20) which describe the evolution of the large-scale fields, it is necessary to calculate the following correlators: 


$$
\begin{aligned}
& T^{31}=\overline{w_{0} u_{0}}=\overline{w_{03}\left(u_{03}\right)^{*}}+\overline{\left(w_{03}\right)^{*} u_{03}} \\
& T^{32}=\overline{w_{0} v_{0}}=\overline{w_{01}\left(v_{01}\right)^{*}}+\overline{\left(w_{01}\right)^{*} v_{01}} \\
& S^{31}=\overline{\widetilde{w}_{0} \tilde{u}_{0}}=\overline{\tilde{w}_{03}\left(\tilde{u}_{03}\right)^{*}}+\overline{\left(\tilde{w}_{03}\right)^{*} \tilde{u}_{03}} \\
& S^{32}=\overline{\widetilde{w}_{0} \tilde{v}_{0}}=\overline{\widetilde{w}_{01}\left(\tilde{v}_{01}\right)^{*}}+\overline{\left(\tilde{w}_{01}\right)^{*} \tilde{v}_{01}} \\
& G^{13}=\overline{u_{0} \tilde{w}_{0}}=\overline{u_{03}\left(\tilde{w}_{03}\right)^{*}}+\overline{\left(u_{03}\right)^{*} \tilde{w}_{03}} \\
& G^{31}=\overline{w_{0} \tilde{u}_{0}}=\overline{w_{03}\left(\tilde{u}_{03}\right)^{*}}+\overline{\left(w_{03}\right)^{*} \tilde{u}_{03}} \\
& G^{23}=\overline{v_{0} \widetilde{w}_{0}}=\overline{v_{01}\left(\tilde{w}_{01}\right)^{*}}+\overline{\left(v_{01}\right)^{*} \tilde{w}_{01}} \\
& G^{32}=\overline{w_{0} \tilde{v}_{0}}=\overline{w_{01}\left(\tilde{v}_{01}\right)^{*}}+\overline{\left(w_{01}\right)^{*} \tilde{v}_{01}}
\end{aligned}
$$

At first let us calculate the Reynolds stresses (96)-(97). For this purpose we use the expressions for the small-scale velocity fields (89)-(91). Their substitution into (96)-(97) gives:

$$
\begin{aligned}
T^{31} & =\frac{f_{0}^{2}}{2} \frac{D_{2} q_{2}}{\left|\hat{A}_{2} \widehat{B}_{2}+D_{2}^{2}\right|^{2}}, \\
T^{32} & =-\frac{f_{0}^{2}}{2} \frac{D_{1} q_{1}}{\left|\widehat{A}_{1} \widehat{B}_{1}+D_{1}^{2}\right|^{2}},
\end{aligned}
$$

where

$$
q_{1,2}=1+\frac{Q H_{1,2}\left(H_{1,2}+\bar{B}_{1,2}\right)}{1+P m^{2}\left(1-W_{1,2}\right)^{2}}-\frac{R a}{1+\operatorname{Pr}^{2}\left(1-W_{1,2}\right)^{2}} .
$$

To calculate the correlators of the magnetic field or the Maxwell stresses $S^{31}$ and $S^{32}$, we use the expressions (92)(94). By substituting (92)-(94) into (98)-(99) we obtain :

$$
S^{31}=\frac{H_{2}^{2}}{\left|\widehat{D}_{H_{2}}\right|^{2}} T^{31}, \quad S^{32}=\frac{H_{1}^{2}}{\left|\widehat{D}_{H_{1}}\right|^{2}} T^{32}
$$

The differences $T^{31}-\widetilde{Q} S^{31}$ and $T^{32}-\widetilde{Q} S^{32}$ contained in the right sides of Eqs. (17)-(18) can be easily found using the expressions (105)-(106):

$$
\begin{aligned}
& T^{31}-\widetilde{Q} S^{31}=T^{31}\left(1-\frac{Q\left(H_{2}+\bar{B}_{2}\right)^{2}}{P m\left|\widehat{D}_{H_{2}}\right|^{2}}\right)=T^{31} Q_{2} \\
& T^{32}-\widetilde{Q} S^{32}=T^{32}\left(1-\frac{Q\left(H_{1}+\bar{B}_{1}\right)^{2}}{P m\left|\widehat{D}_{H_{1}}\right|^{2}}\right)=T^{32} Q_{1}
\end{aligned}
$$

To calculate the group of oscillators (100)-(103) we will use the expressions for the small-scale velocity field (89)-(91) and the magnetic field (92)-(94). Simple mathematical operations yield: 


$$
\begin{aligned}
& G^{13}=\frac{f_{0}^{2}}{4} \frac{i\left(H_{2}+\bar{B}_{2}\right) D_{2}}{\left|\hat{A}_{2} \widehat{B}_{2}+D_{2}^{2}\right|^{2}} \cdot\left(\frac{\widehat{B}_{2}}{\widehat{D}_{H_{2}}^{*}}-\frac{\widehat{B}_{2}^{*}}{\widehat{D}_{H_{2}}}\right) \\
& G^{31}=\frac{f_{0}^{2}}{4} \frac{i\left(H_{2}+\bar{B}_{2}\right) D_{2}}{\left|\widehat{A}_{2} \widehat{B}_{2}+D_{2}^{2}\right|^{2}} \cdot\left(\frac{\widehat{B}_{2}^{*}}{\widehat{D}_{H_{2}}^{*}}-\frac{\widehat{B}_{2}}{\widehat{D}_{H_{2}}}\right) \\
& G^{23}=\frac{f_{0}^{2}}{4} \frac{i\left(H_{1}+\bar{B}_{1}\right) D_{1}}{\left|\hat{A}_{1} \widehat{B}_{1}+D_{1}^{2}\right|^{2}} \cdot\left(\frac{\widehat{B}_{1}^{*}}{\widehat{D}_{H_{1}}}-\frac{\widehat{B}_{1}}{\widehat{D}_{H_{1}}^{*}}\right) \\
& G^{32}=\frac{f_{0}^{2}}{4} \frac{i\left(H_{1}+\bar{B}_{1}\right) D_{1}}{\left|\hat{A}_{1} \widehat{B}_{1}+D_{1}^{2}\right|^{2}} \cdot\left(\frac{\widehat{B}_{1}}{\widehat{D}_{H_{1}}}-\frac{\widehat{B}_{1}^{*}}{\widehat{D}_{H_{1}}^{*}}\right)
\end{aligned}
$$

To close the equations for the large-scale magnetic field (24)-(25), it is necessary to calculate the differences $G^{13}-G^{31}$ and $G^{23}-G^{32}$ corresponding to the turbulent e.m.f. components $\mathcal{E}_{2}=\mathcal{E}_{y}$ and $\mathcal{E}_{1}=\mathcal{E}_{x}$. Taking into account the expressions (109)-(112) we obtain:

$$
\begin{aligned}
& \mathcal{E}_{2}=G^{13}-G^{31}=\frac{f_{0}^{2}}{4} \frac{i\left(H_{2}+\bar{B}_{2}\right) D_{2}}{\left|\widehat{A}_{2} \widehat{B}_{2}+D_{2}^{2}\right|^{2}} \cdot \frac{\left(\widehat{B}_{2}-\widehat{B}_{2}^{*}\right)}{\left|\widehat{D}_{H_{2}}\right|^{2}} \cdot\left(\widehat{D}_{H_{2}}^{*}+\widehat{D}_{H_{2}}\right) \\
& \mathcal{E}_{1}=G^{23}-G^{32}=-\frac{f_{0}^{2}}{4} \frac{i\left(H_{1}+\bar{B}_{1}\right) D_{1}}{\left|\widehat{A}_{1} \widehat{B}_{1}+D_{1}^{2}\right|^{2}} \cdot \frac{\left(\widehat{B}_{1}-\widehat{B}_{1}^{*}\right)}{\left|\widehat{D}_{H_{1}}\right|^{2}} \cdot\left(\widehat{D}_{H_{1}}^{*}+\widehat{D}_{H_{1}}\right)
\end{aligned}
$$

Using the expressions (86) and (92) let us write down some useful formulas:

$$
\begin{aligned}
& \left|\hat{A}_{1,2} \widehat{B}_{1,2}+D_{1,2}^{2}\right|^{2}=\left|\hat{A}_{1,2}\right|^{2} \cdot\left|\widehat{B}_{1,2}\right|^{2}+D_{1,2}^{2} \cdot\left(\hat{A}_{1,2}^{*} \hat{B}_{1,2}^{*}+\hat{A}_{1,2} \hat{B}_{1,2}\right)+D_{1,2}^{4}, \\
& \left|\widehat{A}_{1,2}\right|^{2}=\left|\widehat{D}_{W_{1,2}}\right|^{2}+\widetilde{Q} H_{1,2}\left(H_{1,2}+\bar{B}_{1,2}\right)\left(\frac{\widehat{D}_{W_{1,2}}}{\widehat{D}_{H_{1,2}}^{*}}+\frac{\widehat{D}_{W_{1,2}}^{*}}{\widehat{D}_{H_{1,2}}}\right)+\frac{\widetilde{Q}^{2} H_{1,2}^{2}\left(H_{1,2}+\bar{B}_{1,2}\right)^{2}}{\left|\widehat{D}_{H_{1,2}}\right|^{2}}, \\
& \left|\widehat{B}_{1,2}\right|^{2}=\left|\widehat{A}_{1,2}\right|^{2}-\widetilde{R a}\left(\frac{\hat{A}_{1,2}}{\widehat{D}_{\theta_{1,2}}^{*}}+\frac{\widehat{A}_{1,2}^{*}}{\widehat{D}_{\theta_{1,2}}}\right)+\frac{\widetilde{R a}^{2}}{\left|\widehat{D}_{\theta_{1,2}}\right|^{2}}, \\
& \widehat{A}_{1,2}^{*} \widehat{B}_{1,2}^{*}+\widehat{A}_{1,2} \widehat{B}_{1,2}=\left(\hat{A}_{1,2}^{*}\right)^{2}+\left(\hat{A}_{1,2}\right)^{2}-\widetilde{R a}\left(\frac{\hat{A}_{1,2}^{*}}{\widehat{D}_{\theta_{1,2}}^{*}}+\frac{\hat{A}_{1,2}}{\widehat{D}_{\theta_{1,2}}}\right), \\
& \left|\widehat{D}_{W_{1,2}}\right|^{2}=1+\left(1-W_{1,2}\right)^{2},\left|\widehat{D}_{H_{1,2}}\right|^{2}=P m^{-2}+\left(1-W_{1,2}\right)^{2},\left|\widehat{D}_{\theta_{1,2}}\right|^{2}=P r^{-2}+\left(1-W_{1,2}\right)^{2} \text {, } \\
& \widehat{D}_{H_{1,2}}+\widehat{D}_{H_{1,2}}^{*}=2 P m^{-1}, \widehat{D}_{W_{1,2}} \widehat{D}_{H_{1,2}}+\widehat{D}_{W_{1,2}}^{*} \widehat{D}_{H_{1,2}}^{*}=2\left(P m^{-1}-\left(1-W_{1,2}\right)^{2}\right), \\
& \widehat{D}_{\theta_{1,2}} \widehat{D}_{H_{1,2}}^{*}+\widehat{D}_{H_{1,2}} \widehat{D}_{\theta_{1,2}}^{*}=2\left(\operatorname{Pm}^{-1} \operatorname{Pr}^{-1}+\left(1-W_{1,2}\right)^{2}\right),\left(\widehat{D}_{W_{1,2}}\right)^{2}+\left(\widehat{D}_{W_{1,2}}^{*}\right)^{2}=2\left(1-\left(1-W_{1,2}\right)^{2}\right) \text {, }
\end{aligned}
$$




$$
\widehat{D}_{W_{1,2}}^{*} \widehat{D}_{\theta_{1,2}}+\widehat{D}_{W_{1,2}} \widehat{D}_{\theta_{1,2}}^{*}=2\left(\operatorname{Pr}^{-1}+\left(1-W_{1,2}\right)^{2}\right), \widehat{D}_{W_{1,2}}^{*} \widehat{D}_{H_{1,2}}+\widehat{D}_{W_{1,2}} \widehat{D}_{H_{1,2}}^{*}=2\left(P m^{-1}+\left(1-W_{1,2}\right)^{2}\right) .
$$

Let us substitute these relations into (107)-(108). So, we can find the difference of the Reynolds and Maxwell stresses:

$$
\begin{aligned}
& T^{31}-\widetilde{Q} S^{31}=\frac{f_{0}^{2}}{2} \cdot \frac{D_{2} q_{2} Q_{2}}{4\left(1-W_{2}\right)^{2} q_{2}^{2} \widetilde{Q}_{2}^{2}+\left[D_{2}^{2}+W_{2}\left(2-W_{2}\right)+\mu_{2}\right]^{2}+\xi_{2}}, \\
& T^{32}-\widetilde{Q} S^{32}=\frac{f_{0}^{2}}{2} \cdot \frac{D_{1} q_{1} Q_{1}}{4\left(1-W_{1}\right)^{2} q_{1}^{2} \widetilde{Q}_{1}^{2}+\left[D_{1}^{2}+W_{1}\left(2-W_{1}\right)+\mu_{1}\right]^{2}+\xi_{1}},
\end{aligned}
$$

with the following denotations:

$$
\begin{aligned}
& q_{1,2}=1+\frac{Q H_{1,2}\left(H_{1,2}+\bar{B}_{1,2}\right)}{1+P m^{2}\left(1-W_{1,2}\right)^{2}}-\frac{R a}{1+\operatorname{Pr}^{2}\left(1-W_{1,2}\right)^{2}}, \quad Q_{1,2}=1-\frac{Q P m\left(H_{1,2}+\bar{B}_{1,2}\right)^{2}}{1+P m^{2}\left(1-W_{1,2}\right)^{2}}, \\
& \widetilde{Q}_{1,2}=1-\frac{Q P m H_{1,2}\left(H_{1,2}+\bar{B}_{1,2}\right)}{1+\operatorname{Pm}^{2}\left(1-W_{1,2}\right)^{2}}+\frac{\operatorname{RaPr}}{1+\operatorname{Pr}^{2}\left(1-W_{1,2}\right)^{2}}, \\
& \mu_{1,2}=2 Q H_{1,2}\left(H_{1,2}+\bar{B}_{1,2}\right) \cdot \frac{1+P m\left(1-W_{1,2}\right)^{2}}{1+\operatorname{Pm}^{2}\left(1-W_{1,2}\right)^{2}}+Q^{2} H_{1,2}^{2}\left(H_{1,2}+\bar{B}_{1,2}\right)^{2} \cdot \frac{1-P m^{2}\left(1-W_{1,2}\right)^{2}}{\left(1+P m^{2}\left(1-W_{1,2}\right)^{2}\right)^{2}}- \\
& -R a \cdot \frac{1+\operatorname{Pr}\left(1-W_{1,2}\right)^{2}+2 Q H_{1,2}\left(H_{1,2}+\bar{B}_{1,2}\right) \cdot \frac{1-\operatorname{PrPm}\left(1-W_{1,2}\right)^{2}}{1+\operatorname{Pm}^{2}\left(1-W_{1,2}\right)^{2}}}{1+\operatorname{Pr}^{2}\left(1-W_{1,2}\right)^{2}} \\
& \xi_{1,2}=2 \Xi_{1,2}+2\left(1-W_{1,2}\right)^{2} \Pi_{1,2}-2\left(1-W_{1,2}\right)^{2}\left(1-\widetilde{Q}_{1,2}^{2}\right) \Pi_{1,2}-2\left(1-q_{1,2}^{2}\right) \Xi_{1,2}+\Xi_{1,2} \Pi_{1,2}+ \\
& +\chi_{1,2}\left(1-W_{1,2}\right)^{2}+\chi_{1,2}\left(1+\sigma_{1,2}\right) \\
& \Xi_{1,2}=-\frac{4\left(1-W_{1,2}\right)^{2} \widetilde{Q}_{1,2} \operatorname{RaPr}}{1+\operatorname{Pr}^{2}\left(1-W_{1,2}\right)^{2}}+\frac{2\left(1-W_{1,2}\right)^{2} \operatorname{Ra}^{2} \operatorname{Pr}^{2}}{\left(1+\operatorname{Pr}^{2}\left(1-W_{1,2}\right)^{2}\right)^{2}}+ \\
& +R a \cdot \frac{1+\operatorname{Pr}\left(1-W_{1,2}\right)^{2}+2 Q H_{1,2}\left(H_{1,2}+\bar{B}_{1,2}\right) \cdot \frac{1-\operatorname{PrPm}\left(1-W_{1,2}\right)^{2}}{1+\operatorname{Pm}^{2}\left(1-W_{1,2}\right)^{2}}}{1+\operatorname{Pr}^{2}\left(1-W_{1,2}\right)^{2}} \\
& \Pi_{1,2}=\frac{4 q_{1,2} R a}{1+\operatorname{Pr}^{2}\left(1-W_{1,2}\right)^{2}}+\frac{2 R a^{2}}{\left(1+\operatorname{Pr}^{2}\left(1-W_{1,2}\right)^{2}\right)^{2}}- \\
& -R a \cdot \frac{1+\operatorname{Pr}\left(1-W_{1,2}\right)^{2}+2 Q H_{1,2}\left(H_{1,2}+\bar{B}_{1,2}\right) \cdot \frac{1-\operatorname{Pr} P m\left(1-W_{1,2}\right)^{2}}{1+\operatorname{Pm}^{2}\left(1-W_{1,2}\right)^{2}}}{1+\operatorname{Pr}^{2}\left(1-W_{1,2}\right)^{2}}
\end{aligned}
$$




$$
\begin{gathered}
\sigma_{1,2}=\frac{Q H_{1,2}\left(H_{1,2}+\bar{B}_{1,2}\right)}{1+\operatorname{Pm}^{2}\left(1-W_{1,2}\right)^{2}} \cdot\left[2\left(1+\operatorname{Pm}^{2}\left(1-W_{1,2}\right)^{2}\right)+Q H_{1,2}\left(H_{1,2}+\bar{B}_{1,2}\right)\right] \\
\chi_{1,2}=\frac{2 R a}{1+\operatorname{Pr}^{2}\left(1-W_{1,2}\right)^{2}} \cdot\left[\frac{R a}{2}-\left(1-\operatorname{Pr}\left(1-W_{1,2}\right)^{2}+\right.\right. \\
\left.\left.+\frac{Q H_{1,2}\left(H_{1,2}+\bar{B}_{1,2}\right)\left(1+\operatorname{PrPm}\left(1-W_{1,2}\right)^{2}\right)}{1+\operatorname{Pm}^{2}\left(1-W_{1,2}\right)^{2}}\right)\right]
\end{gathered}
$$

By substituting the relations (115) into (113)-(114) we find the expressions for a turbulent e.m.f. $\mathcal{E}_{1,2}$ in the explicit form:

$$
\begin{aligned}
& \mathcal{E}_{1}=f_{0}^{2} \cdot \frac{D_{1}\left(1-W_{1}\right) P m \widetilde{Q}_{1}\left(H_{1}+\bar{B}_{1}\right)}{\left(1+P m^{2}\left(1-W_{1}\right)^{2}\right)\left[4\left(1-W_{1}\right)^{2} q_{1}^{2} \widetilde{Q}_{1}^{2}+\left[D_{1}^{2}+W_{1}\left(2-W_{1}\right)+\mu_{1}\right]^{2}+\xi_{1}\right]}, \\
& \mathcal{E}_{2}=f_{0}^{2} \cdot \frac{D_{2}\left(1-W_{2}\right) P m \widetilde{Q}_{2}\left(H_{2}+\bar{B}_{2}\right)}{\left(1+P m^{2}\left(1-W_{2}\right)^{2}\right)\left[4\left(1-W_{2}\right)^{2} q_{2}^{2} \widetilde{Q}_{2}^{2}+\left[D_{2}^{2}+W_{2}\left(2-W_{2}\right)+\mu_{2}\right]^{2}+\xi_{2}\right]}
\end{aligned}
$$

\section{ORCID IDs}

(D) Michael I. Kopp https://orcid.org/0000-0001-7457-3272, (DAnatoly V. Tur https://orcid.org/0000-0002-3889-8130, (D) Volodymyr V. Yanovsky https://orcid.org/0000-0003-0461-749X

\section{REFERENCES}

[1] J. Larmor, Rep. Brit. Assoc. Adv. Sc., 159-160 (1919).

[2] G. Moffat, Возбуждение магнитного поля в проводящей среде [Magnetic Field Generation in Electrically Conducting Fluids], (Mir, Moscow, 1980), 343 p. (in Russian)

[3] Ya. Zeldovich, A. Ruzmaikin and D. Sokoloff, Magnetic Fields in Astrophysics, (Gordon and Breach, New York, 1983), pp. 265.

[4] G. Rüdiger, R. Hollerbach, The magnetic universe. Geophysical and astrophysical dynamo theory, (Wiley-VCH Verlag GmbH, Weinheim, 2004), pp. 338.

[5] Chris A. Jones, Dynamo theory (University of Leeds, UK, 2007), pp. 90.

[6] J. Parker, Conversations on Electric and Magnetic Fields in the Cosmos, (Princeton University Press, Princeton, 2007 ), pp. 200.

[7] F. Krauze and K.H. Redler, Магнитная гидродинамика средних полей и теория динамо [Меап-Field Magnetohydrodynamics and Dynamo Theory], (Mir, Moscow, 1984), 314 p. (in Russian)

[8] A. Ruzmaikin, A. Shukurov and D. Sokoloff, Magnetic Fields of Galaxies, (Kluwer, Dordrecht, 1988).

[9] D.D. Sokoloff, R.A. Stepanov, P.G. Frick, Physics-Uspekhi 184 (3), 313-335 (2014), https://doi.org/10.3367/UFNr.0184.201403g.0313.

[10] V.I. Arnold, Ya.B. Zeldovich, A.A. Ruzmalkin, D.D. Sokolov, Sov. Phys. JETP 54(6), 1083-1085 (1981).

[11] V.I. Arnold and B.A. Khesin, Topological Methods in Hydrodynamics, (Springer-Verlag, New York, 1998$)$, pp. 374.

[12] H.P. Greenspan, The theory of Rotating Fluids, (Cambridge At the University Press, 1968), pp. 328.

[13] P.H. Roberts and A.M. Soward, Rotating Fluids in Geophysics, (Academic Press, 1978).

[14] J. Pedlosky, Geophysical Fluid Dynamics (Springer-Verlag, New York, 1987).

[15] V.I. Petviashvili and O.A. Pokhotelov, Solitary Waves in Plasma and Atmosphere, (Gordon\&Breach Science Publishers, 1992).

[16] G.D. Aburjania, Kh.Z. Chargazia and O.A. Kharshiladze, Journal of Atmospheric and Solar-Terrestrial Physics, 72, 971-981 (2010), https://doi.org/10.1016/j.jastp.2010.05.008.2010.

[17] M.Ya. Marov and A.V. Kolesnichenko, Mechanics of Turbulence of Multicomponent Gases (Astrophys. and Space Sci. Library, Vol. 269), (Kluwer Acad. Publ., Dordrecht, 2001), pp. 375.

[18] O.G. Onishchenko, O.A. Pokhotelov and N.M. Astafieva, Physics-Uspekhi, 51(6), 577-590 (2008), http://dx.doi.org/10.1070/PU2008v051n06ABEH006588.

[19] M.V. Nezlin and E.N. Snezhkin, Rossby Vortices and Solitons in Free Motion, (Springer, Berlin, Heidelberg, 1993), pp. 223.

[20] H.K. Moffatt, J. Fluid Mech. 35, 117-129 (1969), https://doi.org/10.1017/S0022112069000991.

[21] M. Steenbeck, F. Krause and K.H. Rädler, Z. Naturforsch, 21a, 369-376 (1966).

[22] H.K. Moffatt, J. Fluid Mech. 106, 27-47 (1981), https://doi.org/10.1017/S002211208100150X.

[23] F. Krause and G. Rüdiger, Astron. Nachr. 295, 93-99 (1974).

[24] S.S. Moiseev, R.Z. Sagdeev, A.V. Tur, G.A. Khomenko and V.V. Yanovsky, Sov. Phys. JETP, 58, 1149-1153 (1983).

[25] S.S. Moiseev, P.B. Rutkevitch, A.V. Tur and V.V. Yanovsky, Sov. Phys. JETP, 67, 294-299 (1988).

[26] E.A. Lypyan, A.A. Mazurov, P.B. Rutkevitch and A.V. Tur, Sov. Phys. JETP, 75, 838-841 (1992).

[27] S.S. Moiseev, R.Z. Sagdeev, A.V. Tur, G.A. Khomenko and A.M. Shukurov, Sov. Phys. Dokl. 28, 925-928 (1983). 
[28] G.V. Levina, S.S. Moiseev and P. B. Rutkevitch, Advances in Fluid Mechanics, 25, 111-161 (2000).

[29] G.V. Levina, M.V. Starkov, S.E. Startsev, V.D. Zimin and S.S. Moiseev, Nonlinear Processes in Geophysics, 7, 49-58 (2000), https://doi.org/10.5194/npg-7-49-2000.

[30] A.V. Tur, V.V. Yanovsky, e-print arXiv: https://arxiv.org/abs/1204.5024v1.

[31] A.V. Tur and V.V. Yanovsky, Open Journal of Fluid Dynamics, 3, 64-74 (2013), https://doi.org/10.4236/ojfd.2013.32009.

[32] P.B. Rutkevich, JETP, 77, 933-938 (1993).

[33] L.M. Smith and F. Waleffe, Physics of Fluids, 11(6), 1608-1622 (1999), https://doi.org/10.1063/1.870022.

[34] L.M. Smith and F. Waleffe, Journal of Fluid Mechanics, 451, 145-168 (2002).

[35] Yu.A. Berezin, V.P. Zhukov, G.V. Levina, S.S. Moiseev, P.B. Rutkevich and A.V. Tur, Heat Transfer-Soviet Research, 21(2), 181-188 (1989).

[36] L.L. Kitchatinov, G. Rüdiger and G. Khomenko, Astron. Astrophys. 287, 320-324 (1994). [37] N. Kleeorin, I. Rogachevskii, https://arxiv.org/abs/1801.00493v1.

[38] U. Frishe, Z.S. She and P.L. Sulem, Physica D, 28, 382-392 (1987), https://doi.org/10.1016/0167-2789(87)90026-1.

[39] V.V. Pipin, G. Rüdiger and L.L. Kitchatinov, Geophys. Astrophys. Fluid Dyn. 83(1), 119-133 (1996), https://doi.org/10.1080/03091929608213644.

[40] O.A. Druzhinin and G.A. Khomenko, in: Nonlinear World, edited by V.G. Baryakhtar (World Scientific, Singapore, 1989), pp. 470.

[41] P.B. Rutkevitch, R.Z. Sagdeev, A.V. Tur and V.V. Yanovsky, in: Proceeding of the IV Intern. Workshop on Nonlinear and Turb. Pros. in Physics (Naukova dumka, Kiev, 1989).

[42] M.I. Kopp, A.V. Tur and V.V. Yanovsky, JETP, 120(4), 733-750 (2015), https://doi.org/10.1134/S1063776115040081.

[43] M.I. Kopp, A.V. Tur and V.V. Yanovsky, https://arxiv.org/abs/1612.08860v1.

[44] P.N. Brandt, G.B. Scharmert, S. Ferguson, R.A. Shine, T.D. Tarbell and A.M. Title, Nature, 335, 238-240 (1988).

[45] O.G. Chkhetiani, S.S. Moiseev and E. Golbraikh, JETP, 87(3), 513-517 (1998), https://doi.org/10.1134/1.558688.

[46] M.I. Kopp, A.V. Tur and V.V Yanovsky, Open Journal of Fluid Dynamics, 05(04), 311-321 (2015), https://doi.org/10.4236/ojfd.2015.54032.

[47] M.I. Kopp, A.V. Tur and V.V Yanovsky, https://arxiv.org/abs/1711.08623v1.

[48] G.Z. Gershuni and E.M. Zhukhovitckii, Convective Stability of Incompressible Fluids (Nauka, Moscow, 1972), pp. 392 (in Russian)

[49] S. Chandrasekhar, Hydrodynamics and Hydromagnetic Stability (Oxford Uni. Press, London, 1961), pp. 652.

[50] M.I. Kopp, A.V. Tur and V.V. Yanovsky, https://arxiv.org/abs/1706.00223v1.

[51] G. Rüdiger, Astron. Nachr. 299(4), 217-222 (1978).

[52] Yu.L. Bolotin, A.V. Tur and V.V. Yanovsky, Chaos: Concepts, Control and Constructive Use (Series: Understanding Complex Systems, Springer, 2016).

\title{
НЕЛІНІЙНЕ ДИНАМО В СТРАТИФІКОВАНІЙ ЕЛЕКТРОПРОВІДНІЙ РІДИНІ, ЩО ПОХИЛО ОБЕРТАЕТЬСЯ В ОДНОРІДНОМУ МАГНІТНОМУ ПОЛІ \\ Михайло Й. Копп ${ }^{\mathrm{a}}$, Анатолій В. Тур ${ }^{\mathrm{c}}$, Костянтин М. Кулика, Володимир В. Яновський \\ ${ }^{a}$ Інститут монокристалів, Національна Академія Наук Украӥни пр. Науки 60, 61001 Харків, Україна \\ ${ }^{b}$ Харківський начіональний університет імені В.Н. Каразина майдан Свободи, 4, 61022, Харків, Украӥна \\ c Університет Тулузи, Інститут астрофізичних досліджень та планетології проспект полковника Роше 9, ВР 44346, 31028, Тулуза Седех 4, Франиія
}

В роботі ми досліджували нову великомасштабну нестійкість, яка виникає в конвективній електропровідній рідині, що похило обертається в зовнішньому однорідному магнітному полі з дрібномасштабною зовнішньою силою, яка має нульову спіральність. Ця сила збуджує дрібномасштабні осциляції швидкості з малим числом Рейнольдса. За допомогою методу багатомасштабних асимптотичних розкладів отримані нелінійні рівняння для вихрових і магнітних збурень в третьому порядку за числом Рейнольдса. Показано, що в результаті спільної дії сили Коріоліса і дрібномасштабної зовнішньої сили в електропровідній рідині, що обертається, можлива великомасштабна нестійкість. Досліджена лінійна стадія магнітовихрового динамо, що виникає в результаті нестійкостей типу $\alpha$-ефекту. Вивчено механізм посилення великомасштабних вихрових збурень внаслідок розвитку гідродинамічного $\alpha$ - ефекту з урахуванням температурної стратифікації середовища. Показано, що «слабке» зовнішне магнітне поле сприяє генерації вихрових та магнітних великомасштабних збурень, а «сильне» зовнішне магнітне поле пригнічує генерацію магніто-вихрових збурень. Чисельними методами знайдені стаціонарні рішення рівнянь нелінійного магніто-вихрового динамо у вигляді локалізованих хаотичних структур у двох випадках, коли немає зовнішнього однорідного магнітного поля і коли воно присутнє.

КЛЮЧОВІ СЛОВА: рівняння магнітної гідродинаміки в наближенні Буссінеска, сила Коріоліса, багатомасштабні асимптотичні розкладання, дрібномасштабна неспіральна турбулентність, $\alpha$-ефект, хаотичні структури.

\author{
НЕЛИНЕЙНОЕ ДИНАМО В НАКЛОННО ВРАЩАЮЩЕЙСЯ СТРАТИФИЦИРОВАННОЙ \\ ЭЛЕКТРОПРОВОДЯЩЕЙ ЖИДКОСТИ В ОДНОРОДНОМ МАГНИТНОМ ПОЛЕ \\ Михаил И. Копп ${ }^{\mathrm{a}}$, Анатолий В. Тур ${ }^{\mathrm{c}}$ Константин Н. Кулика ${ }^{\mathrm{a}}$, Владимир В. Яновский ${ }^{\mathrm{a}, \mathrm{b}}$ \\ аинститут монокристаллов, Начиональная Академия Наук Украины \\ пр. Науки 60, 61001 Харьков, Украина \\ ${ }^{b}$ Харьковский национальный университет имени В.Н. Каразина \\ пл. Свободыл, 4, 61022, Харьков, Украина \\ c Университет Тулузы, Институт астрофизических исследований и планетологии \\ проспект полковника Роше 9, ВР 44346, 31028, Тулуза Сеdех 4, Франция
}


В работе мы исследовали новую крупномасштабную неустойчивость, которая возникает в наклонно вращающейся конвективной электропроводящей жидкости во внешнем однородном магнитном поле с мелкомасштабной внешней силой, имеющей нулевую спиральность. Эта сила возбуждает мелкомасштабные осцилляции скорости с малым числом Рейнольдса. С помощью метода многомасштабных асимптотических разложений получены нелинейные уравнения для вихревых и магнитных возмущений в третьем порядке по числу Рейнольдса. Показано, что в результате совместного действия силы Кориолиса и мелкомасштабной внешней силы во вращающейся электропроводящей жидкости возможна крупномасштабная неустойчивость. Исследована линейная стадия магнито-вихревого динамо, возникающего в результате неустойчивостей типа $\alpha$-эффекта. Изучен механизм усиления крупномасштабных вихревых возмущений, вследствии развития гидродинамического $\alpha$ - эффекта с учетом температурной стратификации среды. Показано, что «слабое» внешнее магнитное поле способствует генерации вихревых и магнитных крупномасштабных возмущений, а «сильное» внешнее магнитное поле подавляет генерацию магнито-вихревых возмущений. Численными методами найдены стационарные решения уравнений нелинейного магнито-вихревого динамо в виде локализованных хаотических структур в двух случаях, когда нет внешнего однородного магнитного поля и когда оно присутствует.

КЛЮЧЕВЫЕ СЛОВА: уравнения магнитной гидродинамики в приближении Буссинеска, сила Кориолиса, многомасштабные асимптотические разложения, мелкомасштабная неспиральная турбулентность, $\alpha$-эффект, хаотические структуры. 